\title{
Rigorous Computation of Smooth Branches of Equilibria for the Three Dimensional Cahn-Hilliard Equation
}

\author{
Marcio Gameiro* Jean-Philippe Lessard ${ }^{\dagger}$
}

September 26, 2010

\begin{abstract}
In this paper, we propose a new general method to compute rigorously global smooth branches of equilibria of higher-dimensional partial differential equations. The theoretical framework is based on a combination of the theory introduced in Global smooth solution curves using rigorous branch following [2] and in Analytic estimates and rigorous continuation for equilibria of higher-dimensional PDEs [11]. Using this method, one can obtain proofs of existence of global smooth solution curves of equilibria for large (continuous) parameter ranges and about local uniqueness of the solutions on the curve. As an application, we compute several smooth branches of equilibria for the three-dimensional Cahn-Hilliard equation.
\end{abstract}

\section{Introduction}

In the last twenty years, various computational methods have been introduced to prove the existence of solutions of nonlinear partial differential equations (PDEs). Among them, methods involving rigorous enclosure of eigenvalues of nonlinear operators are used in $[3,22]$ to prove existence of solutions of second-order elliptic boundary value problems. In [25, 26], a technique of self-consistent a priori bounds is elaborated to prove the existence of equilibria and periodic solutions for the one-dimensional Kuramoto-Sivashinsky PDE. A method based on a computational version of the Banach fixed point theorem is introduced in [24] to prove existence and local uniqueness of solutions of boundary value problems. In [19, 20], a technique combining the Schauder fixed point theorem and a priori error estimates for finite element approximations in Sobolev spaces is applied to prove the existence of solutions of elliptic problems. A combination of norm estimations of inverses of linear operators, Newton-Chord iterations and a priori estimates is developed in [13] to prove existence of steady state solutions of the two-dimensional Navier-Stokes equations. Finally, rigorous numerics combined with ideas from Conley index theory and connection matrices are used in $[9,17]$ to prove results concerning the global dynamics of parabolic PDEs.

*Department of Mathematics, Kyoto University, Kyoto, 606-8502, Japan (gameiro@math.kyoto-u.ac.jp). This author was partially supported by the JSPS Postdoctoral Fellowship No. P08016 and by the JSPS Grant-in-Aid for Scientific Research No. 2008016, Ministry of Education, Science, Technology, Culture and Sports, Japan.

${ }^{\dagger}$ BCAM - Basque Center for Applied Mathematics, Bizkaia Technology Park, 48160 Derio, Bizkaia, Spain and Rutgers University, Department of Mathematics, 110 Frelinghuysen Rd, Piscataway, NJ 08854, USA (lessard@math.rutgers.edu). This author was partially supported by NSF grant DMS-0511115, by DARPA, and by DOE grant DE-FG02-05ER25711. 
These novel computational methods are powerful in the sense that they can prove results that would be difficult to prove using standard analytic techniques. However, their applicability can be limited by the computational time and memory required to terminate the algorithms carrying out the proofs. For example, the computer-assisted proof presented in [15] about the existence of non-trivial bifurcating equilibria for three-dimensional RayleighBénard problems demonstrates how expensive such a technique can be. As the authors mentioned in the conclusion of [15], due to the limit of their computational power, only a few equilibrium solutions could be proved to exist. That raises the question of computational efficiency and applicability of these techniques to higher-dimensional PDEs. The rigorous continuation method developed in $[1,2,10,11,12,16]$ was precisely introduced to address these kind of computational efficiency issues.

In this paper, we propose an extension of the above mentioned rigorous continuation method to the context of global smooth branches of equilibria of higher-dimensional PDEs. In $[9,18,21,23]$, different methods were developed to compute continuous solution curves of one- and two-dimensional PDEs. However, to the best of our knowledge, these techniques have never been applied to equations defined on domains of dimension greater than two. Our new proposed method combines the theory of rigorous branch following introduced in [2] and the analytic estimates constructed in [11]. Using this method, one can obtain proofs of existence of equilibria for continuous parameter ranges, existence and smoothness of global solution curves and about local uniqueness of the solutions on the curves. It is also important to note that although we restrict our attention to the three-dimensional CahnHilliard equation, the method introduced in this paper is quite general and can in principle be applied to a rather large class of nonlinear equations, namely autonomous problems with polynomial nonlinearities posed on rectangular domains.

Consider the three-dimensional Cahn-Hilliard equation

$$
\begin{cases}u_{t}=-\Delta\left(\varepsilon^{2} \Delta u+u-u^{3}\right), & \text { in } \Omega \\ \frac{\partial u}{\partial \nu}=\frac{\partial \Delta u}{\partial \nu}=0, & \text { on } \partial \Omega\end{cases}
$$

defined on a rectangular domain $\Omega \subset \mathbb{R}^{3}$. Here, $\varepsilon>0$ models the interaction length, and $\nu$ denotes the unit outer normal to $\partial \Omega$, that is, there are no-flux boundary conditions for both $u$ and $\Delta u$. Equation (1) was introduced in $[5,6,7]$ as a model for phase separation in binary alloys. Notice that the equilibria of (1) are given by the solutions of the elliptic boundary value problem

$$
\begin{cases}\varepsilon^{2} \Delta u+u-u^{3}=c, & \text { in } \Omega \\ \frac{\partial u}{\partial \nu}=0, & \text { on } \partial \Omega\end{cases}
$$

which introduces the parameter

$$
c:=\frac{1}{|\Omega|} \int_{\Omega}\left(u-u^{3}\right) d y .
$$

In this paper we consider the case $c=0$. The case $c \neq 0$ can also be handled using the theory presented in this paper (see Remark 2.1). We have the following result, which is proved in Section 4.

Theorem 1.1. Consider the case $c=0$ and the three-dimensional rectangular domain $\Omega=[0, \pi] \times[0, \pi / 1.1] \times[0, \pi / 1.2]$. For each segment of curve in the diagram of Figure 1 there exists a smooth branch of equilibria of (1). In particular this implies existence and local uniqueness of solutions, as well as non-existence of secondary bifurcations along the computed branches. 


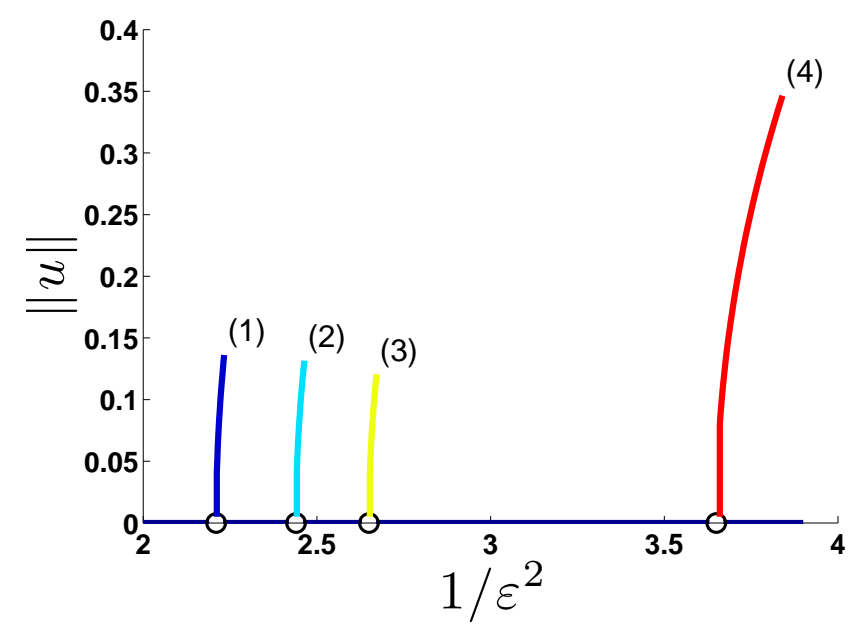

Figure 1: Theorem 1.1 demonstrates the existence of four distinct smooth branches (1), (2), (3) and (4) of equilibrium solutions for the Cahn-Hilliard equation in the 3D domain $\Omega=[0, \pi] \times[0, \pi / 1.1] \times[0, \pi / 1.2]$. Each solution is proved to lie in a ball, given by (10), of radius $r \approx 10^{-12}$ around the computed numerical approximation. Note that the apparent bifurcations from the trivial solution are not proved. The proof of existence and smoothness for each of the branches starts shortly after the bifurcation. More precisely, the bifurcations from the trivial solution occur at $\lambda=1 / \epsilon^{2} \approx 2.21,2.44,2.65$, and 3.65, and the proof of existence and smoothness for the branches (1), (2), (3) and (4) are performed for the parameter intervals $\Lambda^{(1)}=[2.21199048168627,2.23324590395188], \Lambda^{(2)}=$ $[2.44219762780765,2.46395636078611], \Lambda^{(3)}=[2.65238677722770,2.67180897706867]$, and $\Lambda^{(4)}=[3.65987085537112,3.84043126483459]$, respectively. 

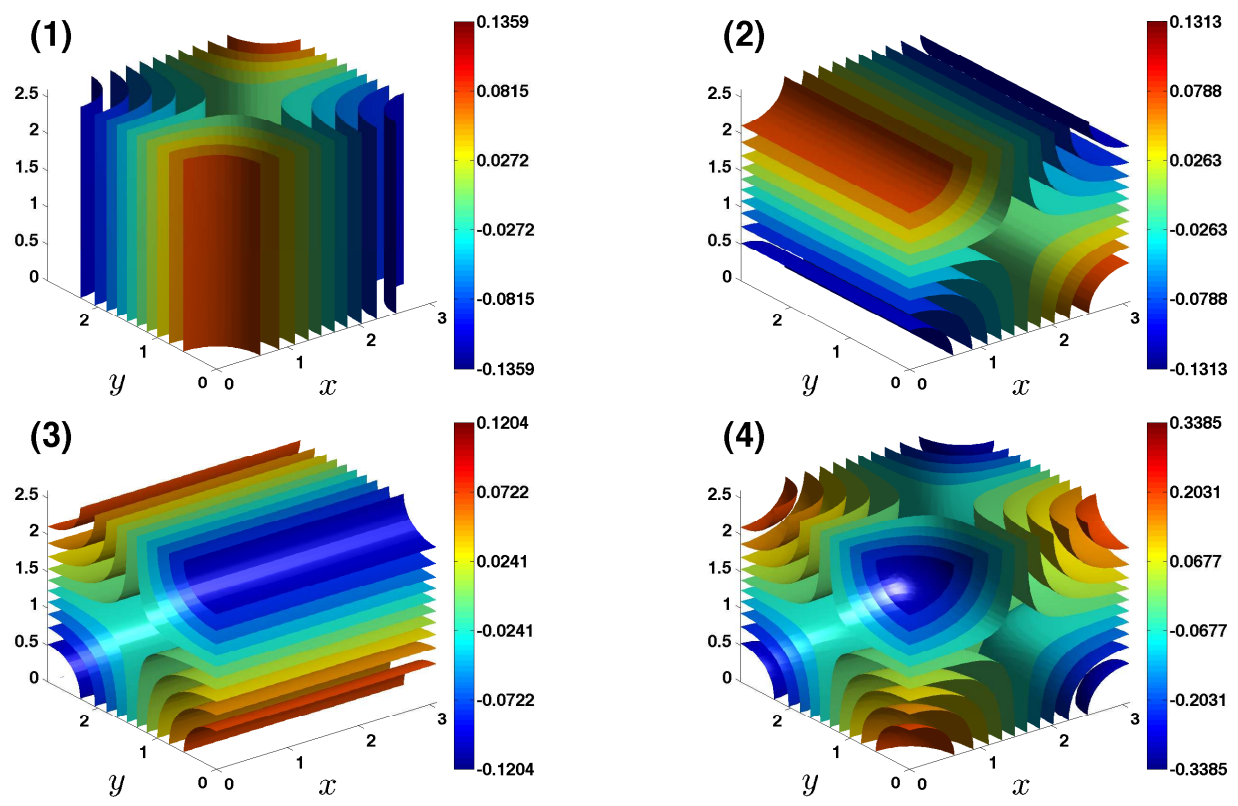

Figure 2: Stationary solutions for the Cahn-Hilliard equation posed on the three-dimensional rectangular domain $\Omega=[0, \pi] \times[0, \pi / 1.1] \times[0, \pi / 1.2]$. Plotted are the isosurfaces of the solutions corresponding to the last point of each branch in the diagram of Figure 1. The labels (1), (2), (3) and (4) correspond to the respective branches in Figure 1. 
The paper is organized as follows. In Section 2, we introduce the general theory of computation of global smooth branches of equilibria of higher-dimensional PDEs with polynomial nonlinearities. In Section 3 we present explicit formulas for the case of a cubic nonlinearity. In Section 4 we apply the method to the three-dimensional Cahn-Hilliard equation, where we prove Theorem 1.1. Note that as a test case we first applied the method to the two-dimensional Cahn-Hilliard equation. These results are presented in Appendix A.

\section{Global smooth branch of equilibria: general theory}

As mentioned in Section 1, we introduce the theory for nonlinear higher-dimensional partial differential equations with polynomial nonlinearities. More precisely, consider the problem of looking for equilibria of the parameter dependent equation

$$
u_{t}=L(u, \lambda)+\sum_{n=2}^{p} q_{n} u^{n}=0,
$$

in a rectangular domain $\Omega \subset \mathbb{R}^{d}$, where $\lambda \in \mathbb{R}$ is a parameter, $L(\cdot, \lambda)$ is a linear operator, $q_{n}=q_{n}(\lambda) \in \mathbb{R}$ are the coefficients of the polynomial nonlinearity of degree $p$. Expanding (4) using an orthogonal basis chosen appropriately in terms of the eigenfunctions of $L(\cdot, \lambda)$ and the boundary conditions, it can be shown that solving problem (4) is equivalent to solving for the zeroes of a problem of the form

$$
f: X^{s} \times \mathbb{R} \rightarrow X^{s}:(x, \lambda) \mapsto f(x, \lambda),
$$

for $x=\left\{x_{\boldsymbol{k}}\right\}_{\boldsymbol{k} \in \mathbb{Z}^{d}}$ in a Banach space $X^{\boldsymbol{s}}$ of fast decaying coefficients, where $\boldsymbol{s}=\left(s_{1}, \ldots, s_{d}\right)$ corresponds to the component-wise decay rate of the coefficients (see [11] for details). More precisely, the nonlinear operator $f=\left\{f_{\boldsymbol{k}}\right\}_{\boldsymbol{k} \in \mathbb{Z}^{d}}$ given by (5) is given component-wise by

$$
f_{\boldsymbol{k}}(x, \lambda):=\mu_{\boldsymbol{k}}(\lambda) x_{\boldsymbol{k}}+\sum_{n=2}^{p} q_{n} \sum_{\substack{\boldsymbol{k}^{1}+\ldots+\boldsymbol{k}^{n}=\boldsymbol{k} \\ \boldsymbol{k}^{j} \in \mathbb{Z}^{d}}} x_{\boldsymbol{k}^{1}} \cdots x_{\boldsymbol{k}^{n}}, \quad \boldsymbol{k}=\left(k_{1}, \ldots, k_{d}\right) \in \mathbb{Z}^{d} .
$$

Remark 2.1. If equation (4) contains a non zero constant $c$, that is if

$$
L(u, \lambda)+\sum_{n=2}^{p} q_{n} u^{n}=c,
$$

then the only difference in $f$ given component-wise by (6) is in its first component $f_{\mathbf{0}}$. In this case, one gets

$$
f_{\mathbf{0}}(x, \lambda)=\mu_{\mathbf{0}}(\lambda) x_{\mathbf{0}}+\sum_{n=2}^{p} q_{n} \sum_{\substack{\boldsymbol{k}^{1}+\ldots+\boldsymbol{k}^{n}=\mathbf{0} \\ \boldsymbol{k}^{j} \in \mathbb{Z}^{d}}} x_{\boldsymbol{k}^{1}} \cdots x_{\boldsymbol{k}^{n}}-c .
$$

It is well accepted that one of the most efficient methods for computing solution curves of a continuous parameterized family of maps $f(x, \lambda)=0$ is to use path-following continuation techniques [14]. Recall that this method involves a predictor and a corrector step: given, within a prescribed tolerance, a solution $x_{0}$ at parameter value $\lambda_{0}$, the predictor step produces an approximate solution $\hat{x}_{1}$ at nearby parameter value $\lambda_{1}$, and the corrector step, 
often based on a Newton-like operator, takes $\hat{x}_{1}$ as its input and produces, once again within the prescribed tolerance, a solution $x_{1}$ at $\lambda_{1}$.

The goal of this paper is to generalize this procedure to the context of computing smooth solution curves of equilibria of higher-dimensional partial differential equations. By the above mentioned equivalence, we restrict our attention to the problem $f(x, \lambda)=0$, where $f$ is given by (5). Since the operator $f$ is infinite dimensional, the continuation must first be applied to some finite dimensional approximation. Hence, let us first introduce the finite dimensional Galerkin projection.

We use boldface type to denote multi-indices as in $\boldsymbol{k}=\left(k_{1}, \ldots, k_{d}\right) \in \mathbb{Z}^{d}$. We denote by $|\cdot|$ the component-wise absolute value, that is, $|\boldsymbol{k}|:=\left(\left|k_{1}\right|, \ldots,\left|k_{d}\right|\right)$. Given $\boldsymbol{k}, \boldsymbol{n} \in \mathbb{Z}^{d}$ we also use component-wise inequalities. So that $\boldsymbol{k}<\boldsymbol{n}$, for example, means that $k_{j}<n_{j}$ for all $1 \leq j \leq d$. We use a similar notation for $\boldsymbol{k} \leq \boldsymbol{n}, \boldsymbol{k}>\boldsymbol{n}$, and $\boldsymbol{k} \geq \boldsymbol{n}$. Throughout this paper $\boldsymbol{m}=\left(m_{1}, \ldots, m_{d}\right)$ and $\boldsymbol{M}=\left(M_{1}, \ldots, M_{d}\right)$ are computational parameters such that $\boldsymbol{M} \geq \boldsymbol{m}$, and $M_{j} \geq 6$ for all $1 \leq j \leq d$. Furthermore $\boldsymbol{s}=\left(s_{1}, \ldots, s_{d}\right)$ will always denote the "decay rate", where each $s_{j}$ is the decay rate on the $j$ th-coordinate, and is such that $s_{j} \geq 2$ for all $1 \leq j \leq d$. We also denote the finite set of indices of "sizes" $\boldsymbol{m}$ and $\boldsymbol{M}$ respectively by $\boldsymbol{F}_{\boldsymbol{m}}:=\left\{\boldsymbol{k} \in \mathbb{Z}^{d}|| \boldsymbol{k} \mid<\boldsymbol{m}\right\}$ and $\boldsymbol{F}_{\boldsymbol{M}}:=\left\{\boldsymbol{k} \in \mathbb{Z}^{d}|| \boldsymbol{k} \mid<\boldsymbol{M}\right\}$. Notice that $\boldsymbol{F}_{\boldsymbol{m}}=F_{m_{1}} \times \cdots \times F_{m_{d}}$, where $F_{m_{j}}:=\left\{k_{j} \in \mathbb{Z}|| k_{j} \mid<m_{j}\right\}$. We have a similar decomposition for $\boldsymbol{F}_{\boldsymbol{M}}$. Given $x=\left\{x_{\boldsymbol{k}}\right\}_{\boldsymbol{k} \in \mathbb{Z}^{d}}$ we denote its finite part of size $\boldsymbol{m}$ and its corresponding infinite part respectively by $x_{\boldsymbol{F}_{m}}:=\left\{x_{\boldsymbol{k}}\right\}_{\boldsymbol{k} \in \boldsymbol{F}_{m}}$ and $x_{\boldsymbol{I}_{m}}:=\left\{x_{\boldsymbol{k}}\right\}_{\boldsymbol{k} \notin \boldsymbol{F}_{m}}$.

A Galerkin projection of (5) of dimension $\boldsymbol{m}$ is given by $f^{(\boldsymbol{m})}:=\left\{f_{\boldsymbol{k}}^{(\boldsymbol{m})}\right\}_{\boldsymbol{k} \in \boldsymbol{F}_{\boldsymbol{m}}}$, where $f_{\boldsymbol{k}}^{(\boldsymbol{m})}: \mathbb{R}^{m_{1} \cdots m_{d}} \times \mathbb{R} \rightarrow \mathbb{R}$, is given by

$$
f_{\boldsymbol{k}}^{(\boldsymbol{m})}\left(x_{\boldsymbol{F}_{\boldsymbol{m}}}, \lambda\right):=f_{\boldsymbol{k}}\left(\left(x_{\boldsymbol{F}_{m}}, 0\right), \lambda\right)=\mu_{\boldsymbol{k}} x_{\boldsymbol{k}}+\sum_{n=2}^{p} q_{n} \sum_{\substack{\boldsymbol{k}^{1}+\ldots+\boldsymbol{k}^{n}=\boldsymbol{k} \\ \boldsymbol{k}^{j} \in \boldsymbol{F}_{m}}} x_{\boldsymbol{k}^{1}} \cdots x_{\boldsymbol{k}^{n}},
$$

for $\boldsymbol{k} \in \boldsymbol{F}_{\boldsymbol{m}}$. Now suppose that at the parameter value $\lambda_{0}$, we numerically find $\bar{x}_{\boldsymbol{F}_{\boldsymbol{m}}}$ and $\dot{x}_{\boldsymbol{F}_{m}}$ such that $f^{(\boldsymbol{m})}\left(\bar{x}_{\boldsymbol{F}_{\boldsymbol{m}}}, \lambda_{0}\right) \approx 0$ and $D f^{(\boldsymbol{m})}\left(\bar{x}_{\boldsymbol{F}_{m}}, \lambda_{0}\right) \dot{x}_{\boldsymbol{F}_{\boldsymbol{m}}}+\frac{\partial f^{(\boldsymbol{m})}}{\partial \lambda}\left(\bar{x}_{\boldsymbol{F}_{m}}, \lambda_{0}\right) \approx 0$. Defining $\bar{x}:=\left(\bar{x}_{\boldsymbol{F}_{m}}, 0_{\boldsymbol{I}_{m}}\right)$ and $\dot{x}:=\left(\dot{x}_{\boldsymbol{F}_{m}}, 0_{\boldsymbol{I}_{m}}\right)$ we should have that

$$
f\left(\bar{x}, \lambda_{0}\right) \approx 0 \text { and } D f\left(\bar{x}, \lambda_{0}\right) \dot{x}+\frac{\partial f}{\partial \lambda}\left(\bar{x}, \lambda_{0}\right) \approx 0,
$$

assuming that the Galerkin projection dimension $\boldsymbol{m}$ is taken large enough. Consider $\lambda$ close to $\lambda_{0}$ and define $\Delta_{\lambda}:=\lambda-\lambda_{0}$. Then, using $\bar{x}$ and $\dot{x}$, we define the set of predictors by

$$
x_{\lambda}=\bar{x}+\Delta_{\lambda} \dot{x} .
$$

The nest step is to construct a parameter dependent fixed point equation whose fixed points correspond to the zeros of $f$. For this purpose, assume that the Jacobian matrix $D f^{(\boldsymbol{m})}\left(\bar{x}_{\boldsymbol{F}_{\boldsymbol{m}}}, \lambda_{0}\right)$ is non-singular and let $J_{\boldsymbol{m}}^{-1}$ be a numerical approximation for its inverse. We define the parameter dependent linear operator $J_{\lambda}^{-1}$ on sequence spaces, which acts as an approximation for the inverse of $D f\left(\bar{x}, \lambda_{0}\right)$. More explicitly, the action of $J_{\lambda}^{-1}$ on an element $x=\left\{x_{\boldsymbol{k}}\right\}_{\boldsymbol{k} \in \mathbb{Z}^{d}}$ is defined component-wise by

$$
\left[J_{\lambda}^{-1}(x)\right]_{\boldsymbol{k}}:= \begin{cases}{\left[J_{\boldsymbol{m}}^{-1}\left(x_{\boldsymbol{F}_{m}}\right)\right]_{\boldsymbol{k}},} & \text { if } \boldsymbol{k} \in \boldsymbol{F}_{\boldsymbol{m}} \\ \left(\mu_{\boldsymbol{k}}(\lambda)\right)^{-1} x_{\boldsymbol{k}}, & \text { if } \boldsymbol{k} \notin \boldsymbol{F}_{\boldsymbol{m}} .\end{cases}
$$

Using the above, for $\lambda$ close to $\lambda_{0}$, we define

$$
T_{\lambda}(x):=x-J_{\lambda}^{-1} f(x, \lambda) .
$$


It is important to notice that the finite part of the operator $J_{\lambda}^{-1}$ depends on $\lambda_{0}$ only. We want to uniquely enclose fixed points of $T_{\lambda}$ into closed balls centered at $x_{\lambda}$ in $X^{s}$. Before proceeding further, let us now describe the set $X^{s}$ in details. Letting

$$
\omega_{k}^{s}:= \begin{cases}1, & \text { if } k=0 \\ |k|^{s}, & \text { if } k \neq 0,\end{cases}
$$

we define the $d$-dimensional weights with growth rate $s=\left(s_{1}, \ldots, s_{d}\right)$ by

$$
\omega_{\boldsymbol{k}}^{\boldsymbol{s}}:=\prod_{j=1}^{d} \omega_{k_{j}}^{s_{j}},
$$

which are used to define the norm

$$
\|x\|_{s}=\sup _{\boldsymbol{k} \in \mathbb{Z}^{d}} \omega_{\boldsymbol{k}}^{\boldsymbol{s}}\left|x_{\boldsymbol{k}}\right|
$$

and the Banach space

$$
X^{s}=\left\{x \mid\|x\|_{s}<\infty\right\},
$$

consisting of sequences with algebraically decaying tails according to the rate $s$. The theoretical justification for this choice of Banach space lies is the fact that the solutions of (5) we are looking for have sufficient regularity.

One can easily check that the closed ball of radius $r$ in $X^{s}$, centered at the origin, is given by

$$
B(r):=\prod_{\boldsymbol{k} \in \mathbb{Z}^{d}}\left[-\frac{r}{\omega_{\boldsymbol{k}}^{s}}, \frac{r}{\omega_{\boldsymbol{k}}^{s}}\right] .
$$

The closed ball of radius $r$ centered at $x_{\lambda}$ is

$$
B\left(x_{\lambda}, r\right):=x_{\lambda}+B(r) .
$$

Consider now bounds $Y_{\boldsymbol{k}}$ and $Z_{\boldsymbol{k}}$ for all $\boldsymbol{k} \in \mathbb{Z}^{d}$, such that

$$
\left|\left[T_{\lambda}\left(x_{\lambda}\right)-x_{\lambda}\right]_{k}\right| \leq Y_{\boldsymbol{k}}\left(\left|\Delta_{\lambda}\right|\right)
$$

and

$$
\sup _{b, c \in B(r)}\left|\left[D T_{\lambda}\left(x_{\lambda}+b\right) c\right]_{k}\right| \leq Z_{\boldsymbol{k}}\left(r,\left|\Delta_{\lambda}\right|\right) .
$$

Let us now make the important remark that the bounds $Y_{\boldsymbol{k}}$ and $Z_{\boldsymbol{k}}$ satisfying (11) and (12) can be constructed monotone increasing in $\left|\Delta_{\lambda}\right| \geq 0$. It is also worth mentioning that in practice, the bound $Y_{\boldsymbol{k}}\left(\left|\Delta_{\lambda}\right|\right)$ is chosen so that $Y_{\boldsymbol{k}}\left(\left|\Delta_{\lambda}\right|\right)=0$ for $\boldsymbol{k} \notin \boldsymbol{F}_{\boldsymbol{M}}$. We refer the reader to Section 3.1 for an explicit construction of such $Y_{k}\left(\left|\Delta_{\lambda}\right|\right)$ and $Z_{k}\left(\left|\Delta_{\lambda}\right|\right)$ in the context of cubic nonlinearities.

Lemma 2.2. Consider $\lambda=\lambda_{0}+\Delta_{\lambda}$. If there exists an $r>0$ such that $\|Y+Z\|_{s}<r$, with $Y:=\left\{Y_{\boldsymbol{k}}\right\}_{\boldsymbol{k} \in \mathbb{Z}^{d}}$ and $Z:=\left\{Z_{\boldsymbol{k}}\right\}_{\boldsymbol{k} \in \mathbb{Z}^{d}}$, satisfying (11) and (12), respectively, then $T_{\lambda}$ is a contraction mapping on $B\left(x_{\lambda}, r\right)$ with contraction constant at most $\|Y+Z\|_{s} / r<1$. Furthermore, there is a unique $\tilde{x}_{\lambda} \in B\left(x_{\lambda}, r\right)$ such that $f\left(\tilde{x}_{\lambda}, \lambda\right)=0$, and $\tilde{x}_{\lambda}$ lies in the interior of $B\left(x_{\lambda}, r\right)$.

Proof. See [11]. 
In order to compute the upper bounds $Y_{\boldsymbol{k}}$ and $Z_{\boldsymbol{k}}$ we choose $\boldsymbol{M} \in \mathbb{N}^{d}$ such that $\boldsymbol{M} \geq$ $p(\boldsymbol{m}-1)+1$ component-wise, that is,

$$
M_{j} \geq p\left(m_{j}-1\right)+1
$$

for all $1 \leq j \leq d$, where $p$ is the degree of the polynomial nonlinearity in (4). This component-wise lower bound on the choice of $\boldsymbol{M}$ comes from the fact that one wants to construct $Y_{\boldsymbol{k}}$ such that $Y_{\boldsymbol{k}}=0$ for all $\boldsymbol{k} \notin \boldsymbol{F}_{\boldsymbol{M}}$. We refer to the beginning of Section 3.1 for an explicit example. Rather than giving general formulas for the upper bounds $Y_{\boldsymbol{k}}$ and $Z_{k}$, we opted to show explicitly in Section 3 how to compute them for the case of a cubic nonlinearity. Assume now that there exists a bound $\tilde{Z}_{M}\left(r,\left|\Delta_{\lambda}\right|\right)$ such that one may define for the cases $\boldsymbol{k} \notin \boldsymbol{F}_{\boldsymbol{M}}$

$$
Z_{\boldsymbol{k}}\left(r,\left|\Delta_{\lambda}\right|\right):=\frac{r}{\omega_{\boldsymbol{k}}^{s}} \tilde{Z}_{M}\left(r,\left|\Delta_{\lambda}\right|\right) .
$$

Notice that $\tilde{Z}_{M}$ is independent of $\boldsymbol{k}$. Its construction is presented in Section 3 in the context of partial differential equations with cubic nonlinearities.

Definition 2.3. We define the finite radii polynomials $\left\{p_{\boldsymbol{k}}(r)\right\}_{\boldsymbol{k} \in \boldsymbol{F}_{M}}$ by

$$
p_{\boldsymbol{k}}\left(r,\left|\Delta_{\lambda}\right|\right):=Y_{\boldsymbol{k}}\left(\left|\Delta_{\lambda}\right|\right)+Z_{\boldsymbol{k}}\left(r,\left|\Delta_{\lambda}\right|\right)-\frac{r}{\omega_{\boldsymbol{k}}^{s}},
$$

and the tail radii polynomial by

$$
\tilde{p}_{M}\left(r,\left|\Delta_{\lambda}\right|\right):=\tilde{Z}_{M}\left(r,\left|\Delta_{\lambda}\right|\right)-1 .
$$

Lemma 2.4. Suppose that $f \in C^{\ell}\left(X^{s} \times \mathbb{R}, X^{s}\right), \ell \in\{1,2, \ldots, \infty\}$. If there exist $r>0$ and $\Delta_{\lambda}$ such that $p_{\boldsymbol{k}}\left(r,\left|\Delta_{\lambda}\right|\right)<0$ for all $\boldsymbol{k} \in \boldsymbol{F}_{\boldsymbol{M}}$ and $\tilde{p}_{\boldsymbol{M}}\left(r,\left|\Delta_{\lambda}\right|\right)<0$, then there exists a $C^{\ell}$ function $\tilde{x}:\left[\lambda_{0}-\left|\Delta_{\lambda}\right|, \lambda_{0}+\left|\Delta_{\lambda}\right|\right] \rightarrow X^{s}: \lambda \mapsto \tilde{x}(\lambda)$ such that $f(\tilde{x}(\lambda), \lambda)=0$ for all $\lambda \in\left[\lambda_{0}-\left|\Delta_{\lambda}\right|, \lambda_{0}+\left|\Delta_{\lambda}\right|\right]$. Furthermore, these are the only solutions of $f(x, \lambda)=0$ in the tube $\left\{(x, \lambda)\left|x-x_{\lambda} \in B(r),\right| \lambda-\lambda_{0}|\leq| \Delta_{\lambda} \mid\right\}$.

Proof. For $\boldsymbol{k} \in \boldsymbol{F}_{\boldsymbol{M}}$, notice that $p_{\boldsymbol{k}}\left(r,\left|\Delta_{\lambda}\right|\right)<0$ implies that

$$
\omega_{\boldsymbol{k}}^{\boldsymbol{s}}\left(Y_{\boldsymbol{k}}\left(\left|\Delta_{\lambda}\right|\right)+Z_{\boldsymbol{k}}\left(r,\left|\Delta_{\lambda}\right|\right)\right)<r .
$$

For $\boldsymbol{k} \notin \boldsymbol{F}_{\boldsymbol{M}}$, recall (14) and that $Y_{\boldsymbol{k}}\left(\left|\Delta_{\lambda}\right|\right)=0$. Then, since $\tilde{p}_{\boldsymbol{M}}\left(r,\left|\Delta_{\lambda}\right|\right)<0$, we get that

$$
\omega_{\boldsymbol{k}}^{s}\left(Y_{\boldsymbol{k}}\left(\left|\Delta_{\lambda}\right|\right)+Z_{\boldsymbol{k}}\left(r,\left|\Delta_{\lambda}\right|\right)\right)=\omega_{\boldsymbol{k}}^{s} Z_{\boldsymbol{k}}\left(r,\left|\Delta_{\lambda}\right|\right)=r \tilde{Z}_{M}\left(r,\left|\Delta_{\lambda}\right|\right)<r .
$$

Therefore we have

$$
\|Y+Z\|_{\boldsymbol{s}}=\sup _{\boldsymbol{k} \in \mathbb{Z}^{d}}\left\{\omega_{\boldsymbol{k}}^{\boldsymbol{s}}\left(Y_{\boldsymbol{k}}\left(\left|\Delta_{\lambda}\right|\right)+Z_{\boldsymbol{k}}\left(r,\left|\Delta_{\lambda}\right|\right)\right)\right\}<r .
$$

As mentioned earlier, we chose the radii polynomials monotone increasing in $\left|\Delta_{\lambda}\right| \geq 0$. Hence, existence and uniqueness of a solution $\tilde{x}(\lambda)$ for all $\lambda \in\left[\lambda_{0}-\left|\Delta_{\lambda}\right|, \lambda_{0}+\left|\Delta_{\lambda}\right|\right]$ follows from Lemma 2.2. In particular, for every fixed $\lambda \in\left[\lambda_{0}-\left|\Delta_{\lambda}\right|, \lambda_{0}+\left|\Delta_{\lambda}\right|\right]$, the operator $T_{\lambda}$ maps $B\left(x_{\lambda}, r\right)$ into itself and it is a contraction on $B\left(x_{\lambda}, r\right)$. Then, the operator

$$
\widetilde{T}: B(r) \times\left[\lambda_{0}-\left|\Delta_{\lambda}\right|, \lambda_{0}+\left|\Delta_{\lambda}\right|\right] \rightarrow B(r):(y, \lambda) \mapsto \widetilde{T}(y, \lambda):=T_{\lambda}\left(y+x_{\lambda}\right)-x_{\lambda}
$$

is a uniform contraction on $B(r)$. Since $f \in C^{\ell}\left(X^{\boldsymbol{s}} \times \mathbb{R}, X^{\boldsymbol{s}}\right)$, we have that

$$
\widetilde{T} \in C^{\ell}\left(B(r) \times\left[\lambda_{0}-\left|\Delta_{\lambda}\right|, \lambda_{0}+\left|\Delta_{\lambda}\right|\right], B(r)\right) .
$$

By the Uniform Contraction Principle, see e.g. [8], we conclude that $\tilde{x}(\lambda)$ is a $C^{\ell}$ function of $\lambda \in\left[\lambda_{0}-\left|\Delta_{\lambda}\right|, \lambda_{0}+\left|\Delta_{\lambda}\right|\right]$. 
For the remainder of this section we assume that $\Delta_{\lambda} \geq 0$. The case $\Delta_{\lambda} \leq 0$ can be handled similarly.

After one successful step, based at $(x, \lambda)=\left(\bar{x}_{0}, \lambda_{0}\right)$ with predictor $\bar{x}_{0}+\Delta_{\lambda} \dot{x}_{0}$ and step size $\Delta_{\lambda}$, we find the corrector $\bar{x}_{1}$ at $\lambda=\lambda_{1}=\lambda_{0}+\Delta_{\lambda}$ using a Newton iteration, and we rebuild the radii polynomials, now based at $(x, \lambda)=\left(\bar{x}_{1}, \lambda_{1}\right)$. Suppose now that we have performed two succesful continuation steps, i.e., in both steps we have found radii $r_{0}$ and $r_{1}$, respectively, where the radii polynomials are negative. We thus have two continuous solution graphs over intervals $\left[\lambda_{0}, \lambda_{1}\right]$ and $\left[\lambda_{1}, \lambda_{2}\right]$ : Lemma 2.4 implies the existence of two functions $x^{0}(\lambda)$ and $x^{1}(\lambda)$ of class $C^{\ell}$ such that $\mathcal{C}_{0}:=\left\{\left(\lambda, x^{0}(\lambda)\right) \mid \lambda \in\left[\lambda_{0}, \lambda_{1}\right]\right\}$ and $\mathcal{C}_{1}:=\left\{\left(\lambda, x^{1}(\lambda)\right) \mid \lambda \in\left[\lambda_{1}, \lambda_{2}\right]\right\}$ are smooth branches of solutions of $f(x, \lambda)=0$. The question is to determine whether or not $\mathcal{C}_{0}$ and $\mathcal{C}_{1}$ connect at the parameter value $\lambda_{1}$ to form a smooth continuum of zeros $\mathcal{C}_{0} \cup \mathcal{C}_{1}$. In other words, can we prove that $x^{0}\left(\lambda_{1}\right)=x^{1}\left(\lambda_{1}\right)$ and that the connection is smooth? At the parameter value $\lambda_{1}$, we have two sets enclosing a unique zero namely

$$
B_{0}:=\bar{x}_{0}+\left(\lambda_{1}-\lambda_{0}\right) \dot{x}_{0}+B\left(r_{0}\right),
$$

and

$$
B_{1}:=\bar{x}_{1}+B\left(r_{1}\right) .
$$

We want to prove that the solutions in $B_{0}$ and $B_{1}$ are the same. We return now to the radii polynomials constructed at basepoint $(x, \lambda)=\left(\bar{x}_{1}, \lambda_{1}\right)$, and evaluate them at $\Delta_{\lambda}=0$. Since $p_{\boldsymbol{k}}\left(r_{1}, 0\right)<0$ for all $\boldsymbol{k} \in \boldsymbol{F}_{\boldsymbol{M}}$ and $\tilde{p}_{\boldsymbol{M}}\left(r_{1}, 0\right)<0$, we can find a non empty interval $\mathcal{I}_{0}:=\left[r_{1}^{-}, r_{1}^{+}\right]$strictly containing $r_{1}$ such that, for all $r \in \mathcal{I}_{0}$, one has that $p_{\boldsymbol{k}}(r, 0)<0$ (for all $\left.\boldsymbol{k} \in \boldsymbol{F}_{\boldsymbol{M}}\right)$ and $\tilde{p}_{\boldsymbol{M}}(r, 0)<0$. We now have two additional sets enclosing a unique zero at parameter value $\lambda_{1}$, namely

$$
B_{1^{ \pm}}:=\bar{x}_{1}+B\left(r_{1}^{ \pm}\right) .
$$

The next result is taken verbatim from [2]. For sake of completeness, we repeat the proof here.

Proposition 2.5. If $B_{0} \subset B_{1+}$ or $B_{1-} \subset B_{0}$, then $\mathcal{C}_{0} \cup \mathcal{C}_{1}$ consists of a continuous branch of solutions of $f(x, \lambda)=0$, and $\mathcal{C}_{0} \cap \mathcal{C}_{1}=\left\{\left(\lambda_{1}, x^{0}\left(\lambda_{1}\right)\right)\right\}=\left\{\left(\lambda_{1}, x^{1}\left(\lambda_{1}\right)\right)\right\} \in\left\{\lambda_{1}\right\} \times B_{0} \cap B_{1}$. Moreover, if $T(x, \lambda):=T_{\lambda}(x)$ is of class $C^{\ell}$, then $\mathcal{C}_{0} \cup \mathcal{C}_{1}$ is a $C^{\ell}$ smooth curve.

Proof. For a geometric representation of the proof, we refer to Figure 3 . The sets $B_{1^{-}}, B_{1^{+}}$ and $B_{1}$ all contain a unique zero of $f\left(\cdot, \lambda_{1}\right)$. Since the balls are nested, these zeros are one and the same, namely $x^{1}\left(\lambda_{1}\right)$. Furthermore, $B_{0}$ also contains exactly one zero of $f\left(\cdot, \lambda_{1}\right)$, namely $x^{0}\left(\lambda_{1}\right)$. By hypothesis we have that either $B_{0}$ and $B_{1^{+}}$, or $B_{0}$ and $B_{1^{-}}$are nested, hence $x^{0}\left(\lambda_{1}\right)=x^{1}\left(\lambda_{1}\right)$. This means that $\mathcal{C}_{0} \cup \mathcal{C}_{1}$ consists of a one dimensional continuous branch of zeros of $f$. It remains to prove smoothness at $\lambda=\lambda_{1}$. By Lemma 2.4, $x^{1}(\lambda)$ is a smooth $C^{\ell}$ function on the interval $\left[\lambda_{1}-\Delta_{\lambda}, \lambda_{1}+\Delta_{\lambda}\right]$. Moreover, we assert that $x^{0}(\lambda)$ and $x^{1}(\lambda)$ coincide on $\left[\lambda_{1}-\epsilon, \lambda_{1}\right]$ for $\epsilon>0$ sufficiently small. Namely, $\left(x^{1}\left(\lambda_{1}\right), \lambda_{1}\right)$ lies in the interior of the tube $\left\{(x, \lambda)\left|x-\left(\bar{x}_{1}+\left(\lambda-\lambda_{1}\right) \dot{x}_{1}\right) \in B\left(r_{1}\right),\right| \lambda-\lambda_{1} \mid \leq \Delta_{\lambda},\right\}$, and $\left(x^{1}(\lambda), \lambda\right)$ are the only zeros of $f$ inside this tube. On the other hand, the solution curve $\left(x^{0}(\lambda), \lambda\right)$ must enter the tube for $\lambda$ close to $\lambda_{1}$, since $\left(x^{0}\left(\lambda_{1}\right), \lambda_{1}\right)$ is in the interior. From uniqueness of solutions inside the tube (Lemma 2.4) it follows that indeed $x^{0}(\lambda)$ and $x^{1}(\lambda)$ coincide on $\left[\lambda_{1}-\epsilon, \lambda_{1}\right]$ for $\epsilon>0$ sufficiently small. Hence, we conclude that the union $\mathcal{C}_{0} \cup \mathcal{C}_{1}$ is $C^{\ell}$ smooth.

Remark 2.6. In practice, the hypothesis of Proposition 2.5 are verified as follows. The center points $\bar{x}_{0}+\left(\lambda_{1}-\lambda_{0}\right) \dot{x}_{0}$ of $B_{0}$ and $\bar{x}_{1}$ of $B_{1}$ and $B_{1^{ \pm}}$are computed using the finite 


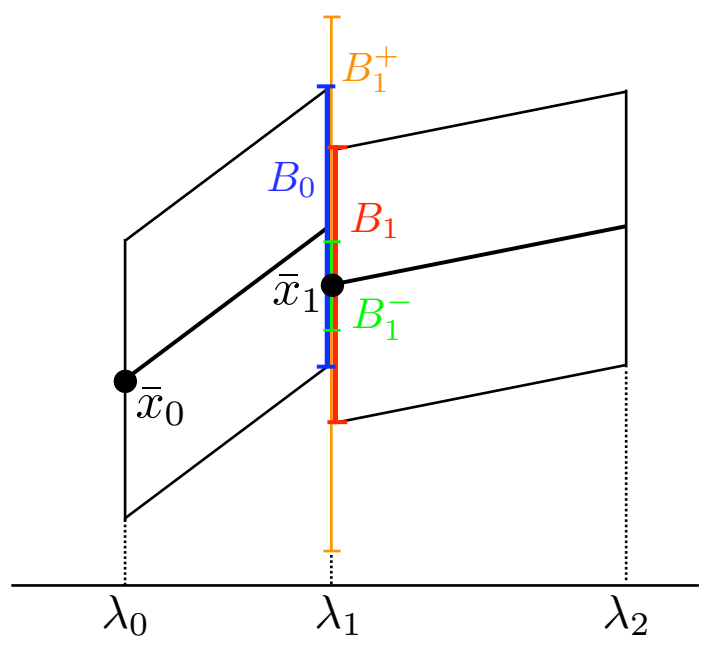

Figure 3: $B_{0} \cap B_{1}$ contains a unique zero of $(5)$ and $\mathcal{C}_{0} \cup \mathcal{C}_{1}$ consists of a continuum of zeros. This picture illustrates the proof of Proposition 2.5

dimensional approximations $f^{\left(\boldsymbol{m}^{0}\right)}$ and $f^{\left(\boldsymbol{m}^{1}\right)}$ of $f$, respectively, where $f^{(\boldsymbol{m})}$ is given by (7). For $\boldsymbol{m}^{0}=\left(m_{1}^{0}, m_{2}^{0}, \ldots, m_{d}^{0}\right)$ and $\boldsymbol{m}^{1}=\left(m_{1}^{1}, m_{2}^{1}, \ldots, m_{d}^{1}\right)$, we define $\overline{\boldsymbol{m}}=\left(\bar{m}_{1}, \bar{m}_{2}, \ldots, \bar{m}_{d}\right)$ component-wise by $\bar{m}_{j}=\max \left\{m_{j}^{0}, m_{j}^{1}\right\}$, for $j=1, \ldots, d$. Let us define the finite dimensional projections

$$
\begin{aligned}
B_{0}^{(\overline{\boldsymbol{m}})} & :=\left(\bar{x}_{0}+\left(\lambda_{1}-\lambda_{0}\right) \dot{x}_{0}\right)_{\boldsymbol{F}_{\overline{\boldsymbol{m}}}}+\prod_{\boldsymbol{k} \in \boldsymbol{F}_{\bar{m}}}\left[-\frac{r_{0}}{\omega_{\boldsymbol{k}}^{\boldsymbol{s}}}, \frac{r_{0}}{\omega_{\boldsymbol{k}}^{\boldsymbol{s}}}\right], \\
B_{1^{-}}^{(\overline{\boldsymbol{m}})} & :=\left(\bar{x}_{1}\right)_{\boldsymbol{F}_{\overline{\boldsymbol{m}}}}+\prod_{\boldsymbol{k} \in \boldsymbol{F}_{\bar{m}}}\left[-\frac{r_{1}^{-}}{\omega_{\boldsymbol{k}}^{\boldsymbol{s}}}, \frac{r_{1}^{-}}{\omega_{\boldsymbol{k}}^{\boldsymbol{s}}}\right], \\
B_{1^{+}}^{(\overline{\boldsymbol{m}})} & :=\left(\bar{x}_{1}\right)_{\boldsymbol{F}_{\overline{\boldsymbol{m}}}}+\prod_{\boldsymbol{k} \in \boldsymbol{F}_{\bar{m}}}\left[-\frac{r_{1}^{+}}{\omega_{\boldsymbol{k}}^{\boldsymbol{s}}}, \frac{r_{1}^{+}}{\omega_{\boldsymbol{k}}^{\boldsymbol{s}}}\right] .
\end{aligned}
$$

Hence, verifying that $B_{0} \subset B_{1^{+}}$(resp. $B_{1^{-}} \subset B_{0}$ ) is done by checking numerically that the finite dimensional box inclusion $B_{0}^{(\overline{\mathbf{m}})} \subset B_{1^{+}}^{(\overline{\boldsymbol{m}})}$ (resp. $B_{1^{-}}^{(\overline{\boldsymbol{m}})} \subset B_{0}^{(\overline{\boldsymbol{m}})}$ ) is satisfied and that $r_{0} \leq r_{1^{+}}\left(\right.$resp. $\left.r_{1^{-}} \leq r_{0}\right)$.

\section{Radii polynomials for smooth branches of equilibria of higher-dimensional PDEs with cubic nonlinearities}

In this section we derive the formulas for the radii polynomials for the case of a cubic nonlinearity, that is, for $f$ of the form

$$
f_{\boldsymbol{k}}(x, \lambda):=\mu_{\boldsymbol{k}}(\lambda) x_{\boldsymbol{k}}+q_{3} \sum_{\substack{\boldsymbol{k}^{1}+\boldsymbol{k}^{2}+\boldsymbol{k}^{3}=\boldsymbol{k} \\ \boldsymbol{k}^{j} \in \mathbb{Z}^{d}}} x_{\boldsymbol{k}^{1}} x_{\boldsymbol{k}^{2}} x_{\boldsymbol{k}^{3}} .
$$


To simplify the exposition, let us introduce the notation

$$
(a * b * c)_{\boldsymbol{k}}:=\sum_{\substack{\boldsymbol{k}^{1}+\boldsymbol{k}^{2}+\boldsymbol{k}^{3}=\boldsymbol{k} \\ \boldsymbol{k}^{j} \in \mathbb{Z}^{d}}} a_{\boldsymbol{k}^{1}} b_{\boldsymbol{k}^{2}} c_{\boldsymbol{k}^{3}} .
$$

\subsection{The bound $Y_{k}\left(\left|\Delta_{\lambda}\right|\right)$}

The computation of the $Y_{\boldsymbol{k}}\left(\left|\Delta_{\lambda}\right|\right)$ in (11) is done as follows. We have that $T\left(x_{\lambda}\right)-x_{\lambda}=$ $-J_{\lambda}^{-1} f\left(x_{\lambda}, \lambda\right)$. Recall now that we choose $\boldsymbol{M}$ so that it satisfies the component-wise lower bound given by (13). Since $x_{\lambda}$ is such that $\left(x_{\lambda}\right)_{\boldsymbol{k}}=0$ for $\boldsymbol{k} \notin \boldsymbol{F}_{\boldsymbol{m}}$ we get that $f_{\boldsymbol{k}}\left(x_{\lambda}, \lambda\right)=0$ for every $\boldsymbol{k} \notin \boldsymbol{F}_{\boldsymbol{M}}$. Hence, for $\boldsymbol{k} \notin \boldsymbol{F}_{\boldsymbol{M}}$, we set $Y_{\boldsymbol{k}}=0$. For the cases $\boldsymbol{k} \in \boldsymbol{F}_{\boldsymbol{M}}$, let us expand $f\left(x_{\lambda}, \lambda\right)$ as a polynomial in $\Delta_{\lambda}$. Consider $\lambda \in\left[\lambda_{0}-\left|\Delta_{\lambda}\right|, \lambda_{0}+\left|\Delta_{\lambda}\right|\right]$. By Taylor's Theorem, one gets the existence of $\xi=\xi(\lambda) \in\left[\lambda_{0}-\left|\Delta_{\lambda}\right|, \lambda_{0}+\left|\Delta_{\lambda}\right|\right]$ such that

$$
\mu_{\boldsymbol{k}}(\lambda)=\mu_{\boldsymbol{k}}\left(\lambda_{0}\right)+\frac{\partial \mu_{\boldsymbol{k}}}{\partial \lambda}\left(\lambda_{0}\right) \Delta_{\lambda}+\frac{\partial^{2} \mu_{\boldsymbol{k}}}{\partial \lambda^{2}}\left(\lambda_{0}\right) \Delta_{\lambda}^{2}+\frac{\partial^{3} \mu_{\boldsymbol{k}}}{\partial \lambda^{3}}(\xi) \Delta_{\lambda}^{3} .
$$

Hence, recalling that $x_{\lambda}=\bar{x}+\Delta_{\lambda} \dot{x}$, one has that

$$
\begin{aligned}
f_{\boldsymbol{k}}\left(x_{\lambda}, \lambda\right)= & \mu_{\boldsymbol{k}}\left(\lambda_{0}\right) \bar{x}+q_{3}\left(\bar{x}^{3}\right)_{\boldsymbol{k}}+\left[\mu_{\boldsymbol{k}}\left(\lambda_{0}\right) \dot{x}+3 q_{3}\left(\bar{x}^{2} * \dot{x}\right)_{\boldsymbol{k}}+\frac{\partial \mu_{\boldsymbol{k}}}{\partial \lambda}\left(\lambda_{0}\right)\right] \Delta_{\lambda} \\
& +\left(\frac{\partial^{2} \mu_{\boldsymbol{k}}}{\partial \lambda^{2}}\left(\lambda_{0}\right) \Delta_{\lambda}^{2}+\frac{\partial^{3} \mu_{\boldsymbol{k}}}{\partial \lambda^{3}}(\xi) \Delta_{\lambda}^{3}\right)\left(\bar{x}+\Delta_{\lambda} \dot{x}\right)+3 q_{3}\left(\bar{x} * \dot{x}^{2}\right)_{\boldsymbol{k}} \Delta_{\lambda}^{2}+q_{3}\left(\dot{x}^{3}\right)_{\boldsymbol{k}} \Delta_{\lambda}^{3} \\
= & f_{\boldsymbol{k}}\left(\bar{x}, \lambda_{0}\right)+\left[D f\left(\bar{x}, \lambda_{0}\right) \dot{x}+\frac{\partial f}{\partial \lambda}\left(\bar{x}, \lambda_{0}\right)\right]_{\boldsymbol{k}} \Delta_{\lambda}+\left(\frac{\partial^{2} \mu_{\boldsymbol{k}}}{\partial \lambda^{2}}\left(\lambda_{0}\right) \bar{x}_{\boldsymbol{k}}+3 q_{3}\left(\bar{x} * \dot{x}^{2}\right)_{\boldsymbol{k}}\right) \Delta_{\lambda}^{2} \\
& +\left(\frac{\partial^{2} \mu_{\boldsymbol{k}}}{\partial \lambda^{2}}\left(\lambda_{0}\right) \dot{x}_{\boldsymbol{k}}+\frac{\partial^{3} \mu_{\boldsymbol{k}}}{\partial \lambda^{3}}(\xi) \bar{x}_{\boldsymbol{k}}+q_{3}\left(\dot{x}^{3}\right)_{\boldsymbol{k}}\right) \Delta_{\lambda}^{3}+\left(\frac{\partial^{3} \mu_{\boldsymbol{k}}}{\partial \lambda^{3}}(\xi) \dot{x}_{\boldsymbol{k}}\right) \Delta_{\lambda}^{4},
\end{aligned}
$$

where we use the notations $x^{2}=x * x$ and $x^{3}=x * x * x$. Consider $\mu_{\boldsymbol{k}}^{(3)}>0$ such that

$$
\left|\frac{\partial^{3} \mu_{\boldsymbol{k}}}{\partial \lambda^{3}}(\xi)\right| \leq \mu_{\boldsymbol{k}}^{(3)},
$$

for all $\xi \in\left[\lambda_{0}-\left|\Delta_{\lambda}\right|, \lambda_{0}+\left|\Delta_{\lambda}\right|\right]$. Given a vector $x$, let us also define another vector $\beta$ component-wise by

$$
\beta_{\boldsymbol{k}}(x):=\mu_{\boldsymbol{k}}^{(3)}\left|x_{\boldsymbol{k}}\right|
$$

For $\boldsymbol{k} \in \boldsymbol{F}_{\boldsymbol{M}}$, define

$$
\begin{aligned}
y_{\boldsymbol{k}}^{(1)} & =\left[D f\left(\bar{x}, \lambda_{0}\right) \dot{x}+\frac{\partial f}{\partial \lambda}\left(\bar{x}, \lambda_{0}\right)\right]_{\boldsymbol{k}} \\
y_{\boldsymbol{k}}^{(2)} & =\frac{\partial^{2} \mu_{\boldsymbol{k}}}{\partial \lambda^{2}}\left(\lambda_{0}\right) \bar{x}_{\boldsymbol{k}}+3 q_{3}\left(\bar{x} * \dot{x}^{2}\right)_{\boldsymbol{k}} \\
y_{\boldsymbol{k}}^{(3)} & =\frac{\partial^{2} \mu_{\boldsymbol{k}}}{\partial \lambda^{2}}\left(\lambda_{0}\right) \dot{x}_{\boldsymbol{k}}+q_{3}\left(\dot{x}^{3}\right)_{\boldsymbol{k}} .
\end{aligned}
$$

Given $\boldsymbol{k} \in \boldsymbol{F}_{\boldsymbol{M}} \backslash \boldsymbol{F}_{\boldsymbol{m}}$ consider $\mu_{\boldsymbol{k}}^{*}>0$ such that

$$
\left|\mu_{\boldsymbol{k}}(\lambda)\right| \geq \mu_{\boldsymbol{k}}^{*}
$$


for all $\lambda \in\left[\lambda_{0}-\left|\Delta_{\lambda}\right|, \lambda_{0}+\left|\Delta_{\lambda}\right|\right]$. Hence we define the bounds $Y^{(0)}, Y^{(1)}, Y^{(2)}, Y^{(3)}$ and $Y^{(4)}$ as

$$
\begin{aligned}
& Y_{\boldsymbol{k}}^{(0)}:= \begin{cases}{\left[\mid J_{\boldsymbol{m}}^{-1} f^{(\boldsymbol{m})}\left(\bar{x}_{\left.\left.\boldsymbol{F}_{\boldsymbol{m}}, \lambda_{0}\right) \mid\right]_{\boldsymbol{k}},}\right.\right.} & \text { if } \boldsymbol{k} \in \boldsymbol{F}_{\boldsymbol{m}} \\
\frac{1}{\mu_{\boldsymbol{k}}^{*}}\left|f_{\boldsymbol{k}}\left(\bar{x}, \lambda_{0}\right)\right|, & \text { if } \boldsymbol{k} \in \boldsymbol{F}_{\boldsymbol{M}} \backslash \boldsymbol{F}_{\boldsymbol{m}} \\
0, & \text { if } \boldsymbol{k} \notin \boldsymbol{F}_{\boldsymbol{M}}\end{cases} \\
& Y_{\boldsymbol{k}}^{(1)}:= \begin{cases}{\left[\left|J_{\boldsymbol{m}}^{-1} y_{\boldsymbol{F}_{\boldsymbol{m}}}^{(1)}\right|\right]_{\boldsymbol{k}},} & \text { if } \boldsymbol{k} \in \boldsymbol{F}_{\boldsymbol{m}} \\
\frac{1}{\mu_{\boldsymbol{k}}^{*}}\left|y_{\boldsymbol{k}}^{(1)}\right|, & \text { if } \boldsymbol{k} \in \boldsymbol{F}_{\boldsymbol{M}} \backslash \boldsymbol{F}_{\boldsymbol{m}} \\
0, & \text { if } \boldsymbol{k} \notin \boldsymbol{F}_{\boldsymbol{M}}\end{cases} \\
& Y_{\boldsymbol{k}}^{(2)}:= \begin{cases}{\left[\left|J_{\boldsymbol{m}}^{-1} y_{\boldsymbol{F}_{\boldsymbol{m}}}^{(2)}\right|\right]_{\boldsymbol{k}},} & \text { if } \boldsymbol{k} \in \boldsymbol{F}_{\boldsymbol{m}} \\
\frac{1}{\mu_{\boldsymbol{k}}^{*}}\left|y_{\boldsymbol{k}}^{(2)}\right|, & \text { if } \boldsymbol{k} \in \boldsymbol{F}_{\boldsymbol{M}} \backslash \boldsymbol{F}_{\boldsymbol{m}} \\
0, & \text { if } \boldsymbol{k} \notin \boldsymbol{F}_{\boldsymbol{M}}\end{cases}
\end{aligned}
$$

and

$$
\begin{aligned}
Y_{\boldsymbol{k}}^{(3)}:= \begin{cases}{\left[\left|J_{\boldsymbol{m}}^{-1} y_{\boldsymbol{F}_{\boldsymbol{m}}}^{(3)}\right|+\left|J_{\boldsymbol{m}}^{-1}\right| \beta_{\boldsymbol{F}_{\boldsymbol{m}}}(\bar{x})\right]_{\boldsymbol{k}},} & \text { if } \boldsymbol{k} \in \boldsymbol{F}_{\boldsymbol{m}} \\
\frac{1}{\mu_{\boldsymbol{k}}^{*}}\left|y_{\boldsymbol{k}}^{(3)}\right|, & \text { if } \boldsymbol{k} \in \boldsymbol{F}_{\boldsymbol{M}} \backslash \boldsymbol{F}_{\boldsymbol{m}} \\
0, & \text { if } \boldsymbol{k} \notin \boldsymbol{F}_{\boldsymbol{M}} .\end{cases} \\
Y_{\boldsymbol{k}}^{(4)}:= \begin{cases}{\left[\left|J_{\boldsymbol{m}}^{-1}\right| \beta_{\boldsymbol{F}_{\boldsymbol{m}}}(\dot{x})\right]_{\boldsymbol{k}},} & \text { if } \boldsymbol{k} \in \boldsymbol{F}_{\boldsymbol{m}} \\
0, & \text { if } \boldsymbol{k} \in \boldsymbol{F}_{\boldsymbol{M}} \backslash \boldsymbol{F}_{\boldsymbol{m}} \\
0, & \text { if } \boldsymbol{k} \notin \boldsymbol{F}_{\boldsymbol{M}} .\end{cases}
\end{aligned}
$$

Note that by (8), one has that the bounds $Y^{(0)}$ and $Y^{(1)}$ should be very small. Hence, using (24), (25), (26), (27) and (28), one defines

$$
Y\left(\left|\Delta_{\lambda}\right|\right):=\sum_{j=0}^{4} Y^{(j)}\left|\Delta_{\lambda}\right|^{j} .
$$

\subsection{The bound $Z_{k}\left(r,\left|\Delta_{\lambda}\right|\right)$}

In order to compute $Z_{\boldsymbol{k}}$ it is convenient to denote $\tilde{J}_{\boldsymbol{m}}:=D f^{(\boldsymbol{m})}\left(\bar{x}_{\boldsymbol{F}_{\boldsymbol{m}}}, \lambda_{0}\right)$ and introduce the operator

$$
\left[\tilde{J}_{\lambda}(x)\right]_{\boldsymbol{k}}:= \begin{cases}{\left[\tilde{J}_{\boldsymbol{m}}\left(x_{\boldsymbol{F}_{\boldsymbol{m}}}\right)\right]_{\boldsymbol{k}},} & \text { if } \boldsymbol{k} \in \boldsymbol{F}_{\boldsymbol{m}} \\ \mu_{\boldsymbol{k}}(\lambda) x_{\boldsymbol{k}}, & \text { if } \boldsymbol{k} \notin \boldsymbol{F}_{\boldsymbol{m}},\end{cases}
$$

which acts as an approximate inverse for the operator $J_{\lambda}^{-1}$. We consider the splitting

$$
D T_{\lambda}\left(x_{\lambda}+b\right) c=\left(I-J_{\lambda}^{-1} \tilde{J}_{\lambda}\right) c-J_{\lambda}^{-1}\left(D f\left(x_{\lambda}+b, \lambda\right)-\tilde{J}_{\lambda}\right) c,
$$

where the first term is very small for $\boldsymbol{k} \in \boldsymbol{F}_{\boldsymbol{m}}$, and is zero for $\boldsymbol{k} \notin \boldsymbol{F}_{\boldsymbol{m}}$. For $\boldsymbol{k} \in \boldsymbol{F}_{\boldsymbol{m}}$ we have the bounds

$$
\left|\left[\left(I-J_{\lambda}^{-1} \tilde{J}_{\lambda}\right) c\right]_{\boldsymbol{k}}\right| \leq r\left[\left|I-J_{\boldsymbol{m}}^{-1} D f^{(\boldsymbol{m})}\left(\bar{x}_{\boldsymbol{F}_{\boldsymbol{m}}}, \lambda_{0}\right)\right| \omega_{\boldsymbol{F}_{\boldsymbol{m}}}^{-\boldsymbol{s}}\right]_{\boldsymbol{k}}=: r d_{\boldsymbol{k}}^{(1,0)},
$$


where $\omega_{\boldsymbol{F}_{m}}^{-\boldsymbol{s}}:=\left\{1 / \omega_{\boldsymbol{k}}^{\boldsymbol{s}}\right\}_{\boldsymbol{k} \in \boldsymbol{F}_{m}}$, and $|\cdot|$ represents component-wise absolute values. As for the second term in (31), we have that

$$
\left[D f\left(x_{\lambda}+b, \lambda\right) c\right]_{\boldsymbol{k}}=\mu_{\boldsymbol{k}}(\lambda) c_{\boldsymbol{k}}+3 q_{3}\left[x_{\lambda}^{2} * c+2 x_{\lambda} * b * c+b^{2} * c\right]_{\boldsymbol{k}} .
$$

Furthermore, for $\boldsymbol{k} \in \boldsymbol{F}_{\boldsymbol{m}}$,

$$
\left[\tilde{J}_{\lambda} c\right]_{\boldsymbol{k}}=\left[D f^{(\boldsymbol{m})}\left(\bar{x}_{\boldsymbol{F}_{\boldsymbol{m}}}, \lambda_{0}\right) c_{\boldsymbol{F}_{\boldsymbol{m}}}\right]_{\boldsymbol{k}}=\mu_{\boldsymbol{k}}\left(\lambda_{0}\right) c_{\boldsymbol{k}}+3 q_{3} \sum_{\substack{\boldsymbol{k}^{1}+\boldsymbol{k}^{2}+\boldsymbol{k}^{3}=\boldsymbol{k} \\ \boldsymbol{k}^{j} \in \boldsymbol{F}_{m}}} \bar{x}_{\boldsymbol{k}^{1}} \bar{x}_{\boldsymbol{k}^{2}} c_{\boldsymbol{k}^{3}}
$$

and, for $\boldsymbol{k} \notin \boldsymbol{F}_{\boldsymbol{m}}$,

$$
\left[\tilde{J}_{\lambda} c\right]_{\boldsymbol{k}}=\mu_{\boldsymbol{k}}(\lambda) c_{\boldsymbol{k}}
$$

We now consider $u, v \in B(1)$ defined by $b=r u$ and $c=r v$ so that we can expand the expression $\left[\left(D f\left(x_{\lambda}+b, \lambda\right)-\tilde{J}_{\lambda}\right) c\right]_{\boldsymbol{k}}$ in terms of $r$ and $\Delta_{\lambda}$. Considering $\boldsymbol{k} \in \boldsymbol{F}_{\boldsymbol{M}}$, we have that

$$
\left[\left(D f\left(x_{\lambda}+b, \lambda\right)-\tilde{J}_{\lambda}\right) c\right]_{\boldsymbol{k}}= \begin{cases}\left(\mu_{\boldsymbol{k}}(\lambda)-\mu_{\boldsymbol{k}}\left(\lambda_{0}\right)\right) c_{\boldsymbol{k}}+3 q_{3} \sum_{j=1}^{3} \sum_{l=0}^{3-j} C_{\boldsymbol{k}}^{(j, l)} r^{j} \Delta_{\lambda}^{l}, & \text { if } \boldsymbol{k} \in \boldsymbol{F}_{\boldsymbol{m}} \\ 3 q_{3} \sum_{j=1}^{3} \sum_{l=0}^{3-j} C_{\boldsymbol{k}}^{(j, l)} r^{j} \Delta_{\lambda}^{l}, & \text { if } \boldsymbol{k} \in \boldsymbol{F}_{\boldsymbol{M}} \backslash \boldsymbol{F}_{\boldsymbol{m}}\end{cases}
$$

where the coefficients $C_{\boldsymbol{k}}^{(j, l)}$ are given by

$$
\begin{gathered}
C_{\boldsymbol{k}}^{(1,0)}:=\left\{\begin{array}{cl}
\sum_{\substack{\boldsymbol{k}^{1}+\boldsymbol{k}^{2}+\boldsymbol{k}^{3}=\boldsymbol{k} \\
\boldsymbol{k}^{3} \notin \boldsymbol{F}_{m}}} \bar{x}_{\boldsymbol{k}^{1}} \bar{x}_{\boldsymbol{k}^{2}} v_{\boldsymbol{k}^{3}}, \quad \text { if } \boldsymbol{k} \in \boldsymbol{F}_{\boldsymbol{m}} \\
\sum_{\substack{\boldsymbol{k}^{1}+\boldsymbol{k}^{2}+\boldsymbol{k}^{3}=\boldsymbol{k} \\
\boldsymbol{k}^{j} \in \mathbb{Z}^{d}}} \bar{x}_{\boldsymbol{k}^{1}} \bar{x}_{\boldsymbol{k}^{2}} v_{\boldsymbol{k}^{3}}, \quad \text { if } \boldsymbol{k} \in \boldsymbol{F}_{\boldsymbol{M}} \backslash \boldsymbol{F}_{\boldsymbol{m}}
\end{array}\right. \\
C_{\boldsymbol{k}}^{(1,1)}:=\underset{\substack{\boldsymbol{k}^{1}+\boldsymbol{k}^{2}+\boldsymbol{k}^{3}=\boldsymbol{k} \\
\boldsymbol{k}^{j} \in \mathbb{Z}^{d}}}{ } \bar{x}_{\boldsymbol{k}^{1}} \dot{x}_{\boldsymbol{k}^{2}} v_{\boldsymbol{k}^{3}}, \quad C_{\boldsymbol{k}}^{(1,2)}:=\sum_{\substack{\boldsymbol{k}^{1}+\boldsymbol{k}^{2}+\boldsymbol{k}^{3}=\boldsymbol{k} \\
\boldsymbol{k}^{j} \in \mathbb{Z}^{d}}} \dot{x}_{\boldsymbol{k}^{1}} \dot{x}_{\boldsymbol{k}^{2}} v_{\boldsymbol{k}^{3}} \\
C_{\boldsymbol{k}}^{(2,0)}:=\underset{\substack{\boldsymbol{k}^{1}+\boldsymbol{k}^{2}+\boldsymbol{k}^{3}=\boldsymbol{k} \\
\boldsymbol{k}^{j} \in \mathbb{Z}^{d}}}{2} \bar{x}_{\boldsymbol{k}^{1}} u_{\boldsymbol{k}^{2}} v_{\boldsymbol{k}^{3}}, \quad C_{\boldsymbol{k}}^{(2,1)}:=\underset{\substack{\boldsymbol{k}^{1}+\boldsymbol{k}^{2}+\boldsymbol{k}^{3}=\boldsymbol{k} \\
\boldsymbol{k}^{j} \in \mathbb{Z}^{d}}}{2} \dot{x}_{\boldsymbol{k}^{1}} u_{\boldsymbol{k}^{2}} v_{\boldsymbol{k}^{3}},
\end{gathered}
$$

and

$$
C_{\boldsymbol{k}}^{(3,0)}:=\sum_{\substack{\boldsymbol{k}^{1}+\boldsymbol{k}^{2}+\boldsymbol{k}^{3}=\boldsymbol{k} \\ \boldsymbol{k}^{j} \in \mathbb{Z}^{d}}} u_{\boldsymbol{k}^{1}} u_{\boldsymbol{k}^{2}} v_{\boldsymbol{k}^{3}}
$$

We now want to find upper bounds $Z_{\boldsymbol{k}}^{(1,0)}, Z_{\boldsymbol{k}}^{(1,1)}, Z_{\boldsymbol{k}}^{(1,2)}, Z_{\boldsymbol{k}}^{(2,0)}, Z_{\boldsymbol{k}}^{(2,1)}$ and $Z_{\boldsymbol{k}}^{(3,0)}$ so that $\left|C_{\boldsymbol{k}}^{(j, l)}\right| \leq Z_{\boldsymbol{k}}^{(j, l)}$. Consider the splitting

$$
C_{\boldsymbol{k}}^{(1,0)}= \begin{cases}\sum_{\substack{\boldsymbol{k}^{1}+\boldsymbol{k}^{2}+\boldsymbol{k}^{3}=k \\ \boldsymbol{k}^{3} \in \boldsymbol{F}_{M} \backslash \boldsymbol{F}_{m}}} \bar{x}_{\boldsymbol{k}^{1}} \bar{x}_{\boldsymbol{k}^{2}} v_{\boldsymbol{k}^{3}}+\sum_{\substack{\boldsymbol{k}^{1}+\boldsymbol{k}^{2}+\boldsymbol{k}^{3}=\boldsymbol{k} \\ \boldsymbol{k}^{3} \notin \boldsymbol{F}_{M}}} \bar{x}_{\boldsymbol{k}^{1}} \bar{x}_{\boldsymbol{k}^{2}} v_{\boldsymbol{k}^{3}}, & \text { if } \boldsymbol{k} \in \boldsymbol{F}_{\boldsymbol{m}} \\ \sum_{\substack{\boldsymbol{k}^{1}+\boldsymbol{k}^{2}+\boldsymbol{k}^{3}=\boldsymbol{k} \\ \boldsymbol{k}^{j} \in \boldsymbol{F}_{M}}} \bar{x}_{\boldsymbol{k}^{1}} \bar{x}_{\boldsymbol{k}^{2}} v_{\boldsymbol{k}^{3}}+\sum_{\substack{\boldsymbol{k}^{1}+\boldsymbol{k}^{2}+\boldsymbol{k}^{3}=\boldsymbol{k} \\\left\{\boldsymbol{k}^{1}, \boldsymbol{k}^{2}, \boldsymbol{k}^{3}\right\} \not \subset \boldsymbol{F}_{M}}} \bar{x}_{\boldsymbol{k}^{1}} \bar{x}_{\boldsymbol{k}^{2}} v_{\boldsymbol{k}^{3}}, & \text { if } \boldsymbol{k} \notin \boldsymbol{F}_{\boldsymbol{m}} .\end{cases}
$$


Define

$$
\begin{aligned}
\bar{A} & :=\max _{\boldsymbol{k} \in \boldsymbol{F}_{m}}\left\{\omega_{\boldsymbol{k}}^{\boldsymbol{s}}\left|\bar{x}_{\boldsymbol{k}}\right|\right\} \\
\dot{A} & :=\max _{\boldsymbol{k} \in \boldsymbol{F}_{m}}\left\{\omega_{\boldsymbol{k}}^{s}\left|\dot{x}_{\boldsymbol{k}}\right|\right\} .
\end{aligned}
$$

Using Lemma 2.2 from [11] for the case $\boldsymbol{k} \in \boldsymbol{F}_{\boldsymbol{m}}$, and Corollary 3.3 from [11] for the case $\boldsymbol{k} \in \boldsymbol{F}_{\boldsymbol{M}} \backslash \boldsymbol{F}_{\boldsymbol{m}}$, we set

$$
Z_{\boldsymbol{k}}^{(1,0)}:=\left\{\begin{array}{l}
\sum_{\substack{\boldsymbol{k}^{1}+\boldsymbol{k}^{2}+\boldsymbol{k}^{3}=\boldsymbol{k} \\
\boldsymbol{k}^{3} \in \boldsymbol{F}_{M} \backslash \boldsymbol{F}_{m}}}\left|\bar{x}_{\boldsymbol{k}^{1}}\right|\left|\bar{x}_{\boldsymbol{k}^{2}}\right|\left(1 / \omega_{\boldsymbol{k}^{3}}^{\boldsymbol{s}}\right)+\bar{A}^{2} \varepsilon_{\boldsymbol{k}}^{(3)}, \quad \text { if } \boldsymbol{k} \in \boldsymbol{F}_{\boldsymbol{m}} \\
\sum_{\substack{\boldsymbol{k}^{1}+\boldsymbol{k}^{2}+\boldsymbol{k}^{3}=\boldsymbol{k} \\
\boldsymbol{k}^{j} \in \boldsymbol{F}_{M}}}\left|\bar{x}_{\boldsymbol{k}^{1}}\right|\left|\bar{x}_{\boldsymbol{k}^{2}}\right|\left(1 / \omega_{\boldsymbol{k}^{3}}^{\boldsymbol{s}}\right)+3 \bar{A}^{2} \varepsilon_{\boldsymbol{k}}^{(3)}, \quad \text { if } \boldsymbol{k} \in \boldsymbol{F}_{\boldsymbol{M}} \backslash \boldsymbol{F}_{\boldsymbol{m}},
\end{array}\right.
$$

where the definition of $\varepsilon_{\boldsymbol{k}}^{(3)}$ can be found in equation (12) in [11]. For $C_{\boldsymbol{k}}^{(1,1)}, C_{\boldsymbol{k}}^{(1,2)}, C_{\boldsymbol{k}}^{(2,0)}$, $C_{\boldsymbol{k}}^{(2,1)}$ and $C_{\boldsymbol{k}}^{(3,0)}$ we consider the splittings

$$
\begin{aligned}
& C_{\boldsymbol{k}}^{(1,1)}=2 \sum_{\substack{\boldsymbol{k}^{1}+\boldsymbol{k}^{2}+\boldsymbol{k}^{3}=\boldsymbol{k} \\
\boldsymbol{k}^{j} \in \boldsymbol{F}_{M}}} \bar{x}_{\boldsymbol{k}^{1}} \dot{x}_{\boldsymbol{k}^{2}} v_{\boldsymbol{k}^{3}}+2 \sum_{\substack{\boldsymbol{k}^{1}+\boldsymbol{k}^{2}+\boldsymbol{k}^{3}=\boldsymbol{k} \\
\left\{\boldsymbol{k}^{1}, \boldsymbol{k}^{2}, \boldsymbol{k}^{3}\right\} \not \subset \boldsymbol{F}_{M}}} \bar{x}_{\boldsymbol{k}^{1}} \dot{x}_{\boldsymbol{k}^{2}} v_{\boldsymbol{k}^{3}}, \\
& C_{\boldsymbol{k}}^{(1,2)}=\sum_{\substack{\boldsymbol{k}^{1}+\boldsymbol{k}^{2}+\boldsymbol{k}^{3}=\boldsymbol{k} \\
\boldsymbol{k}^{j} \in \boldsymbol{F}_{M}}} \dot{x}_{\boldsymbol{k}^{1}} \dot{x}_{\boldsymbol{k}^{2}} v_{\boldsymbol{k}^{3}}+\sum_{\substack{\boldsymbol{k}^{1}+\boldsymbol{k}^{2}+\boldsymbol{k}^{3}=\boldsymbol{k} \\
\left\{\boldsymbol{k}^{1}, \boldsymbol{k}^{2}, \boldsymbol{k}^{3}\right\} \not \subset \boldsymbol{F}_{M}}} \dot{x}_{\boldsymbol{k}^{1}} \dot{x}_{\boldsymbol{k}^{2}} v_{\boldsymbol{k}^{3}}, \\
& C_{\boldsymbol{k}}^{(2,0)}=2 \sum_{\substack{\boldsymbol{k}^{1}+\boldsymbol{k}^{2}+\boldsymbol{k}^{3}=\boldsymbol{k} \\
\boldsymbol{k}^{j} \in \boldsymbol{F}_{M}}} \bar{x}_{\boldsymbol{k}^{1}} u_{\boldsymbol{k}^{2}} v_{\boldsymbol{k}^{3}}+2 \sum_{\substack{\boldsymbol{k}^{1}+\boldsymbol{k}^{2}+\boldsymbol{k}^{3}=\boldsymbol{k} \\
\left\{\boldsymbol{k}^{1}, \boldsymbol{k}^{2}, \boldsymbol{k}^{3}\right\}} \boldsymbol{F}_{M}} \bar{x}_{\boldsymbol{k}^{1}} u_{\boldsymbol{k}^{2}} v_{\boldsymbol{k}^{3}}, \\
& C_{\boldsymbol{k}}^{(2,1)}=2 \sum_{\substack{\boldsymbol{k}^{1}+\boldsymbol{k}^{2}+\boldsymbol{k}^{3}=\boldsymbol{k} \\
\boldsymbol{k}^{j} \in \boldsymbol{F}_{M}}} \dot{x}_{\boldsymbol{k}^{1}} u_{\boldsymbol{k}^{2}} v_{\boldsymbol{k}^{3}}+2 \sum_{\substack{\boldsymbol{k}^{1}+\boldsymbol{k}^{2}+\boldsymbol{k}^{3}=\boldsymbol{k} \\
\left\{\boldsymbol{k}^{1}, \boldsymbol{k}^{2}, \boldsymbol{k}^{3}\right\} \not \subset \boldsymbol{F}_{M}}} \dot{x}_{\boldsymbol{k}^{1}} u_{\boldsymbol{k}^{2}} v_{\boldsymbol{k}^{3}}, \\
& C_{\boldsymbol{k}}^{(3,0)}=\sum_{\substack{\boldsymbol{k}^{1}+\boldsymbol{k}^{2}+\boldsymbol{k}^{3}=\boldsymbol{k} \\
\boldsymbol{k}^{j} \in \boldsymbol{F}_{M}}} u_{\boldsymbol{k}^{1}} u_{\boldsymbol{k}^{2}} v_{\boldsymbol{k}^{3}}+\sum_{\substack{\boldsymbol{k}^{1}+\boldsymbol{k}^{2}+\boldsymbol{k}^{3}=\boldsymbol{k} \\
\left\{\boldsymbol{k}^{1}, \boldsymbol{k}^{2}, \boldsymbol{k}^{3}\right\} \not \subset \boldsymbol{F}_{M}}} u_{\boldsymbol{k}^{1}} u_{\boldsymbol{k}^{2}} v_{\boldsymbol{k}^{3}} .
\end{aligned}
$$

Recalling (33) and (34), we again use Corollary 3.3 from [11] to set, for $\boldsymbol{k} \in \boldsymbol{F}_{\boldsymbol{M}}$,

$$
\begin{aligned}
Z_{\boldsymbol{k}}^{(1,1)} & =2 \sum_{\substack{\boldsymbol{k}^{1}+\boldsymbol{k}^{2}+\boldsymbol{k}^{3}=\boldsymbol{k} \\
\boldsymbol{k}^{j} \in \boldsymbol{F}_{M}}}\left|\bar{x}_{\boldsymbol{k}^{1}} \| \dot{x}_{\boldsymbol{k}^{2}}\right|\left(1 / \omega_{\boldsymbol{k}^{3}}^{\boldsymbol{s}}\right)+6 \bar{A} \dot{A} \varepsilon_{\boldsymbol{k}}^{(3)}, \\
Z_{\boldsymbol{k}}^{(1,2)}= & \sum_{\substack{\boldsymbol{k}^{1}+\boldsymbol{k}^{2}+\boldsymbol{k}^{3}=\boldsymbol{k} \\
\boldsymbol{k}^{j} \in \boldsymbol{F}_{M}}}\left|\dot{x}_{\boldsymbol{k}^{1}}\right|\left|\dot{x}_{\boldsymbol{k}^{2}}\right|\left(1 / \omega_{\boldsymbol{k}^{3}}^{\boldsymbol{s}}\right)+3 \dot{A}^{2} \varepsilon_{\boldsymbol{k}}^{(3)}, \\
Z_{\boldsymbol{k}}^{(2,0)}= & 2 \sum_{\substack{\boldsymbol{k}^{1}+\boldsymbol{k}^{2}+\boldsymbol{k}^{3}=\boldsymbol{k} \\
\boldsymbol{k}^{j} \in \boldsymbol{F}_{M}}}\left|\bar{x}_{\boldsymbol{k}^{1}}\right|\left(1 / \omega_{\boldsymbol{k}^{2}}^{\boldsymbol{s}}\right)\left(1 / \omega_{\boldsymbol{k}^{3}}^{\boldsymbol{s}}\right)+6 \bar{A} \varepsilon_{\boldsymbol{k}}^{(3)}, \\
Z_{\boldsymbol{k}}^{(2,1)}= & 2 \sum_{\substack{\boldsymbol{k}^{1}+\boldsymbol{k}^{2}+\boldsymbol{k}^{3}=\boldsymbol{k} \\
\boldsymbol{k}^{j} \in \boldsymbol{F}_{M}}}\left|\dot{x}_{\boldsymbol{k}^{1}}\right|\left(1 / \omega_{\boldsymbol{k}^{2}}^{\boldsymbol{s}}\right)\left(1 / \omega_{\boldsymbol{k}^{3}}^{\boldsymbol{s}}\right)+6 \dot{A} \varepsilon_{\boldsymbol{k}}^{(3)}, \\
Z_{\boldsymbol{k}}^{(3,0)}= & \sum_{\substack{\boldsymbol{k}^{1}+\boldsymbol{k}^{2}+\boldsymbol{k}^{3}=\boldsymbol{k} \\
\boldsymbol{k}^{j} \in \boldsymbol{F}_{M}}}\left(1 / \omega_{\boldsymbol{k}^{1}}^{\boldsymbol{s}}\right)\left(1 / \omega_{\boldsymbol{k}^{2}}^{\boldsymbol{s}}\right)\left(1 / \omega_{\boldsymbol{k}^{3}}^{\boldsymbol{s}}\right)+3 \varepsilon_{\boldsymbol{k}}^{(3)},
\end{aligned}
$$


For $\boldsymbol{k} \in \boldsymbol{F}_{\boldsymbol{m}}$, one has that

$$
\left(\mu_{\boldsymbol{k}}(\lambda)-\mu_{\boldsymbol{k}}\left(\lambda_{0}\right)\right) c_{\boldsymbol{k}}=\left(\frac{\partial \mu_{\boldsymbol{k}}}{\partial \lambda}\left(\lambda_{0}\right) \Delta_{\lambda}+\frac{\partial^{2} \mu_{\boldsymbol{k}}}{\partial \lambda^{2}}\left(\lambda_{0}\right) \Delta_{\lambda}^{2}+\frac{\partial^{3} \mu_{\boldsymbol{k}}}{\partial \lambda^{3}}(\xi) \Delta_{\lambda}^{3}\right) c_{\boldsymbol{k}},
$$

hence

$$
\left|\left(\mu_{\boldsymbol{k}}(\lambda)-\mu_{\boldsymbol{k}}\left(\lambda_{0}\right)\right) c_{\boldsymbol{k}}\right| \leq\left(\left|\frac{\partial \mu_{\boldsymbol{k}}}{\partial \lambda}\left(\lambda_{0}\right)\right|\left|\Delta_{\lambda}\right|+\left|\frac{\partial^{2} \mu_{\boldsymbol{k}}}{\partial \lambda^{2}}\left(\lambda_{0}\right)\right|\left|\Delta_{\lambda}\right|^{2}+\left|\frac{\partial^{3} \mu_{\boldsymbol{k}}}{\partial \lambda^{3}}(\xi)\right|\left|\Delta_{\lambda}\right|^{3}\right) \frac{r}{\omega_{\boldsymbol{k}}^{s}} .
$$

For $\boldsymbol{k} \in \boldsymbol{F}_{\boldsymbol{m}}$, define $d_{\boldsymbol{k}}^{(1,1)}, d_{\boldsymbol{k}}^{(1,2)}$ and $d_{\boldsymbol{k}}^{(1,3)}$ by

$$
\begin{aligned}
d_{\boldsymbol{k}}^{(1,1)} & :=\left|\frac{\partial \mu_{\boldsymbol{k}}}{\partial \lambda}\left(\lambda_{0}\right)\right| \frac{1}{\omega_{\boldsymbol{k}}^{\boldsymbol{s}}} \\
d_{\boldsymbol{k}}^{(1,2)} & :=\left|\frac{\partial^{2} \mu_{\boldsymbol{k}}}{\partial \lambda^{2}}\left(\lambda_{0}\right)\right| \frac{1}{\omega_{\boldsymbol{k}}^{\boldsymbol{s}}} \\
d_{\boldsymbol{k}}^{(1,3)} & :=\mu_{\boldsymbol{k}}^{(3)} \frac{1}{\omega_{\boldsymbol{k}}^{\boldsymbol{s}}} .
\end{aligned}
$$

One now has to define the term $\tilde{Z}_{\boldsymbol{M}}\left(r,\left|\Delta_{\lambda}\right|\right)$ from equation (14). For the case $\boldsymbol{k} \notin \boldsymbol{F}_{\boldsymbol{M}}$, one has that

$$
\left[\left(D f\left(x_{\lambda}+b, \lambda\right)-\tilde{J}_{\lambda}\right) c\right]_{\boldsymbol{k}}=3 q_{3} \sum_{j=1}^{3} \sum_{l=0}^{3-j} C_{\boldsymbol{k}}^{(j, l)} r^{j} \Delta_{\lambda}^{l},
$$

where the coefficients $C_{\boldsymbol{k}}^{(j, l)}$ are given by

$$
\begin{aligned}
C_{\boldsymbol{k}}^{(1,0)} & :=\sum_{\substack{\boldsymbol{k}^{1}+\boldsymbol{k}^{2}+\boldsymbol{k}^{3}=\boldsymbol{k} \\
\boldsymbol{k}^{j} \in \mathbb{Z}^{d}}} \bar{x}_{\boldsymbol{k}^{1}} \bar{x}_{\boldsymbol{k}^{2}} v_{\boldsymbol{k}^{3}}, \quad C_{\boldsymbol{k}}^{(1,1)}:=\underset{\substack{\boldsymbol{k}^{1}+\boldsymbol{k}^{2}+\boldsymbol{k}^{3}=\boldsymbol{k} \\
\boldsymbol{k}^{j} \in \mathbb{Z}^{d}}}{2} \bar{x}_{\boldsymbol{k}^{1}} \dot{x}_{\boldsymbol{k}^{2}} v_{\boldsymbol{k}^{3}}, \quad C_{\boldsymbol{k}}^{(1,2)}:=\sum_{\substack{\boldsymbol{k}^{1}+\boldsymbol{k}^{2}+\boldsymbol{k}^{3}=\boldsymbol{k} \\
\boldsymbol{k}^{j} \in \mathbb{Z}^{d}}} \dot{x}_{\boldsymbol{k}^{1}} \dot{x}_{\boldsymbol{k}^{2}} v_{\boldsymbol{k}^{3}}, \\
C_{\boldsymbol{k}}^{(2,0)} & :=\underset{\substack{\boldsymbol{k}^{1}+\boldsymbol{k}^{2}+\boldsymbol{k}^{3}=\boldsymbol{k} \\
\boldsymbol{k}^{j} \in \mathbb{Z}^{d}}}{2} \bar{x}_{\boldsymbol{k}^{1}} u_{\boldsymbol{k}^{2}} v_{\boldsymbol{k}^{3}}, \quad C_{\boldsymbol{k}}^{(2,1)}:=\underset{\substack{\boldsymbol{k}^{1}+\boldsymbol{k}^{2}+\boldsymbol{k}^{3}=\boldsymbol{k} \\
\boldsymbol{k}^{j} \in \mathbb{Z}^{d}}}{2} \sum_{\boldsymbol{k}^{1}} u_{\boldsymbol{k}^{2}} v_{\boldsymbol{k}^{3}}, \quad C_{\boldsymbol{k}}^{(3,0)}:=\sum_{\substack{\boldsymbol{k}^{1}+\boldsymbol{k}^{2}+\boldsymbol{k}^{3}=\boldsymbol{k} \\
\boldsymbol{k}^{j} \in \mathbb{Z}^{d}}} u_{\boldsymbol{k}^{1}} u_{\boldsymbol{k}^{2}} v_{\boldsymbol{k}^{3}} .
\end{aligned}
$$

Assume now that there exists a uniform (in $\boldsymbol{k}$ and $\lambda$ ) lower bound $\tilde{\mu}_{\boldsymbol{M}}$ such that

$$
\left|\mu_{\boldsymbol{k}}(\lambda)\right| \geq \tilde{\mu}_{M}
$$

for all $\boldsymbol{k} \notin \boldsymbol{F}_{\boldsymbol{M}}$ and for all $\lambda \in\left[\lambda_{0}-\left|\Delta_{\lambda}\right|, \lambda_{0}+\left|\Delta_{\lambda}\right|\right]$. One can use Lemma 2.1 from [11] to get that

$$
\begin{aligned}
\left|\left[D T_{\lambda}\left(x_{\lambda}+b\right) c\right]_{\boldsymbol{k}}\right| & =\left|\frac{3 q_{3}}{\mu_{\boldsymbol{k}}} \sum_{j=1}^{3} \sum_{l=0}^{3-j} C_{\boldsymbol{k}}^{(j, l)} r^{j} \Delta_{\lambda}^{l}\right| \\
& \leq \frac{3\left|q_{3}\right| \tilde{\alpha}_{M}^{(3)}}{\tilde{\mu}_{M}}\left(\bar{A}^{2}+2 \bar{A} \dot{A} \Delta_{\lambda}+\dot{A}^{2} \Delta_{\lambda}^{2}+2 \bar{A} r+2 \dot{A} r \Delta_{\lambda}+r^{2}\right) \frac{r}{\omega_{\boldsymbol{k}}^{\boldsymbol{s}}}
\end{aligned}
$$

where the explicit definition of $\tilde{\alpha}_{M}^{(3)}$ can be found in equation (13) in [11]. Defining

$$
\tilde{Z}_{M}\left(r,\left|\Delta_{\lambda}\right|\right)=\frac{3\left|q_{3}\right| \tilde{\alpha}_{M}^{(3)}}{\tilde{\mu}_{M}}\left(\bar{A}^{2}+2 \bar{A} \dot{A} \Delta_{\lambda}+\dot{A}^{2} \Delta_{\lambda}^{2}+2 \bar{A} r+2 \dot{A} r \Delta_{\lambda}+r^{2}\right)
$$


one can finally define

$$
Z_{\boldsymbol{k}}\left(r,\left|\Delta_{\lambda}\right|\right):= \begin{cases}3\left|q_{3}\right|\left[\left|J_{\boldsymbol{m}}^{-1}\right|\left(\sum_{l=1}^{3} d_{\boldsymbol{F}_{\boldsymbol{m}}}^{(1, l)} r\left|\Delta_{\lambda}\right|^{l}+\sum_{j=1}^{3} \sum_{l=0}^{3-j} Z_{\boldsymbol{F}_{\boldsymbol{m}}}^{(j, l)} r^{j}\left|\Delta_{\lambda}\right|^{l}\right)\right]_{\boldsymbol{k}}+d_{\boldsymbol{k}}^{(1,0)} r, & \text { if } \boldsymbol{k} \in \boldsymbol{F}_{\boldsymbol{m}} \\ \frac{3\left|q_{3}\right|}{\mu_{\boldsymbol{k}}^{*}} \sum_{j=1}^{3} \sum_{l=0}^{3-j} Z_{\boldsymbol{k}}^{(j, l)} r^{j}\left|\Delta_{\lambda}\right|^{l}, & \text { if } \boldsymbol{k} \in \boldsymbol{F}_{\boldsymbol{M}} \backslash \boldsymbol{F}_{\boldsymbol{m}} \\ \frac{r}{\omega_{\boldsymbol{k}}^{\boldsymbol{s}}} \tilde{Z}_{\boldsymbol{M}}\left(r,\left|\Delta_{\lambda}\right|\right), & \text { if } \boldsymbol{k} \notin \boldsymbol{F}_{\boldsymbol{M}}\end{cases}
$$

\subsection{Definition of the radii polynomials}

We then have that the radii polynomials, defined in Definition 2.3, for the general cubic problem (17) are given, for $\boldsymbol{k} \in \boldsymbol{F}_{\boldsymbol{m}}$, by

$$
\begin{aligned}
p_{\boldsymbol{k}}\left(r,\left|\Delta_{\lambda}\right|\right)= & \sum_{j=0}^{4} Y_{\boldsymbol{k}}^{(j)}\left|\Delta_{\lambda}\right|^{j}+\left(d_{\boldsymbol{k}}^{(1,0)}+3\left|q_{3}\right|\left[\left|J_{\boldsymbol{m}}^{-1}\right|\left(\sum_{l=1}^{3} d_{\boldsymbol{F}_{\boldsymbol{m}}}^{(1, l)}\left|\Delta_{\lambda}\right|^{l}+\sum_{l=0}^{2} Z_{\boldsymbol{F}_{\boldsymbol{m}}}^{(1, l)}\left|\Delta_{\lambda}\right|^{l}\right)\right]_{\boldsymbol{k}}-1 / \omega_{\boldsymbol{k}}^{\boldsymbol{s}}\right) r \\
& +\left(3\left|q_{3}\right|\left[\left|J_{\boldsymbol{m}}^{-1}\right|\left(Z_{\boldsymbol{F}_{\boldsymbol{m}}^{(2,0)}}+Z_{\boldsymbol{F}_{\boldsymbol{m}}}^{(2,1)}\left|\Delta_{\lambda}\right|\right)\right]_{\boldsymbol{k}}\right) r^{2}+\left(3\left|q_{3}\right|\left[\left|J_{\boldsymbol{m}}^{-1}\right| Z_{\boldsymbol{F}_{\boldsymbol{m}}}^{(3,0)}\right]_{\boldsymbol{k}}\right) r^{3}
\end{aligned}
$$

for $\boldsymbol{k} \in \boldsymbol{F}_{\boldsymbol{M}} \backslash \boldsymbol{F}_{\boldsymbol{m}}$, by

$$
\begin{aligned}
p_{\boldsymbol{k}}\left(r,\left|\Delta_{\lambda}\right|\right)= & \sum_{j=0}^{3} Y_{\boldsymbol{k}}^{(j)}\left|\Delta_{\lambda}\right|^{j}+\left(\frac{3\left|q_{3}\right|}{\mu_{\boldsymbol{k}}^{*}} \sum_{l=0}^{2} Z_{\boldsymbol{k}}^{(1, l)}\left|\Delta_{\lambda}\right|^{l}-\frac{1}{\omega_{\boldsymbol{k}}^{\boldsymbol{s}}}\right) r \\
& +\frac{6\left|q_{3}\right|}{\mu_{\boldsymbol{k}}^{*}}\left(Z_{\boldsymbol{k}}^{(2,0)}+Z_{\boldsymbol{k}}^{(2,1)}\left|\Delta_{\lambda}\right|\right) r^{2}+\frac{3\left|q_{3}\right|}{\mu_{\boldsymbol{k}}^{*}} Z_{\boldsymbol{k}}^{(3,0)} r^{3},
\end{aligned}
$$

and finally,

$$
\tilde{p}_{M}\left(r,\left|\Delta_{\lambda}\right|\right)=\tilde{Z}_{M}\left(r,\left|\Delta_{\lambda}\right|\right)-1 .
$$

\section{Application to the Cahn-Hilliard Equation}

In this section we apply the theory introduced in Section 2 and we use the explicit radii polynomials constructed in Section 3 to the Cahn-Hilliard equation (1), for the case $c=0$ in (2). Taking the domain as

$$
\Omega=\prod_{j=1}^{d}\left[0, \ell_{j}\right],
$$

and recalling the Neumann boundary conditions, we can express the solutions in terms of a cosine basis $\left\{\psi_{\boldsymbol{k}}\right\}_{\boldsymbol{k} \in \mathbb{N}^{d}}$ given by

$$
\psi_{\boldsymbol{k}}(y):=\prod_{j=1}^{d} \cos \left(k_{j} L_{j} y_{j}\right),
$$

where $L_{j}=\pi / \ell_{j}$, for $j=1, \ldots, d$. Notice that we only need to consider the basis elements for $\boldsymbol{k} \geq \mathbf{0}$. However, if we use the expansion

$$
u=\sum_{\boldsymbol{k} \in \mathbb{Z}^{d}} a_{\boldsymbol{k}} \psi_{\boldsymbol{k}}
$$


with the assumption that $a_{|\boldsymbol{k}|}=a_{\boldsymbol{k}}$ for $\boldsymbol{k} \in \mathbb{Z}^{d}$, then the expansion of (2) takes the form

$$
f_{k}(a, \lambda):=\mu_{\boldsymbol{k}} a_{\boldsymbol{k}}-\sum_{\substack{\boldsymbol{k}^{1}+\boldsymbol{k}^{2}+\boldsymbol{k}^{3}=k \\ \boldsymbol{k}^{j} \in \mathbb{Z}^{d}}} a_{\boldsymbol{k}^{1}} a_{\boldsymbol{k}^{2}} a_{\boldsymbol{k}^{3}}
$$

where

$$
\mu_{\boldsymbol{k}}=1-\frac{1}{\lambda}\left(k_{1}^{2} L_{1}^{2}+\cdots+k_{d}^{2} L_{d}^{2}\right),
$$

with $\lambda=1 / \varepsilon^{2}$, and we have that $f_{|\boldsymbol{k}|}=f_{\boldsymbol{k}}$, for all $\boldsymbol{k} \in \mathbb{Z}^{d}$. Therefore, we only need to solve $f_{\boldsymbol{k}}=0$ for $\boldsymbol{k} \geq \mathbf{0}$.

\subsection{Completion of the radii polynomials for Cahn-Hilliard}

To complete the definition of the radii polynomials for the Cahn-Hilliard equation given by (45), (46) and (47), we must compute some quantities specific to (1). To define the bounds $Y_{\boldsymbol{k}}$, one needs to compute $\frac{\partial^{2} \mu_{\boldsymbol{k}}}{\partial \lambda^{2}}\left(\lambda_{0}\right), \mu_{\boldsymbol{k}}^{(3)}$ from (18), $\beta_{\boldsymbol{k}}(\bar{x})$ and $\beta_{\boldsymbol{k}}(\dot{x})$ given by (19), $y_{\boldsymbol{k}}^{(1)}$,

$y_{\boldsymbol{k}}^{(2)}$ and $y_{\boldsymbol{k}}^{(3)}$ given respectively by $(20),(21)$ and (22), and finally $\mu_{\boldsymbol{k}}^{*}$ given by (23). First notice that

$$
\begin{aligned}
\frac{\partial \mu_{\boldsymbol{k}}}{\partial \lambda}\left(\lambda_{0}\right) & =\frac{1}{\lambda_{0}^{2}}\left(k_{1}^{2} L_{1}^{2}+\cdots+k_{d}^{2} L_{d}^{2}\right), \\
\frac{\partial^{2} \mu_{\boldsymbol{k}}}{\partial \lambda^{2}}\left(\lambda_{0}\right) & =-\frac{2}{\lambda_{0}^{3}}\left(k_{1}^{2} L_{1}^{2}+\cdots+k_{d}^{2} L_{d}^{2}\right), \\
\frac{\partial^{3} \mu_{\boldsymbol{k}}}{\partial \lambda^{3}}(\xi) & =\frac{6}{\xi^{4}}\left(k_{1}^{2} L_{1}^{2}+\cdots+k_{d}^{2} L_{d}^{2}\right) .
\end{aligned}
$$

For the construction of the radii polynomials, we assume the extra condition that

$$
\left|\Delta_{\lambda}\right| \leq \frac{\lambda_{0}}{2}
$$

Note that this condition need to be explicitly verified during the computations. Hence, for every $\xi \in\left[\lambda_{0}-\left|\Delta_{\lambda}\right|, \lambda_{0}+\left|\Delta_{\lambda}\right|\right]$,

$$
\left|\frac{\partial^{3} \mu_{\boldsymbol{k}}}{\partial \lambda^{3}}(\xi)\right| \leq \mu_{\boldsymbol{k}}^{(3)}:=\frac{96}{\lambda_{0}^{4}}\left(k_{1}^{2} L_{1}^{2}+\cdots+k_{d}^{2} L_{d}^{2}\right) .
$$

The constructions of the $\beta_{\boldsymbol{k}}(\bar{x}), \beta_{\boldsymbol{k}}(\dot{x}), y_{\boldsymbol{k}}^{(1)}, y_{\boldsymbol{k}}^{(2)}$ and $y_{\boldsymbol{k}}^{(3)}$ are straightforward using (50) and (53). Note that $y_{\boldsymbol{k}}^{(1)}$ is explicitly given by

$$
y_{\boldsymbol{k}}^{(1)}= \begin{cases}\mu_{\boldsymbol{k}}\left(\lambda_{0}\right) \dot{x}_{\boldsymbol{k}}-3\left(\bar{x}^{2} * \dot{x}\right)_{\boldsymbol{k}}+\frac{\partial \mu_{\boldsymbol{k}}}{\partial \lambda}\left(\lambda_{0}\right) \bar{x}_{\boldsymbol{k}}, & \text { if } \boldsymbol{k} \in \boldsymbol{F}_{\boldsymbol{m}} \\ -3\left(\bar{x}^{2} * \dot{x}\right)_{\boldsymbol{k}}, & \text { if } \boldsymbol{k} \in \boldsymbol{F}_{\boldsymbol{M}} \backslash \boldsymbol{F}_{\boldsymbol{m}} .\end{cases}
$$

For the construction of $\mu_{k}^{*}$ we proceed as follows. Recall that we consider $\lambda \in\left[\lambda_{0}-\left|\Delta_{\lambda}\right|, \lambda_{0}+\right.$ $\left.\left|\Delta_{\lambda}\right|\right]$ and that we assume $\left|\Delta_{\lambda}\right| \leq \frac{\lambda_{0}}{2}$. Assume also that

$$
\min _{1 \leq j \leq d}\left\{m_{j}^{2} L_{j}^{2}\right\}>\frac{3 \lambda_{0}}{2} .
$$


Hence, for $\boldsymbol{k} \notin \boldsymbol{F}_{\boldsymbol{m}}$, we get that

$$
\begin{aligned}
\left|\mu_{\boldsymbol{k}}(\lambda)\right| & =\left|1-\frac{1}{\lambda}\left(k_{1}^{2} L_{1}^{2}+\cdots+k_{d}^{2} L_{d}^{2}\right)\right| \\
& =\frac{1}{\lambda}\left(k_{1}^{2} L_{1}^{2}+\cdots+k_{d}^{2} L_{d}^{2}\right)-1 \\
& \geq \frac{2}{3 \lambda_{0}}\left(k_{1}^{2} L_{1}^{2}+\cdots+k_{d}^{2} L_{d}^{2}\right)-1 \\
& \geq \frac{2}{3 \lambda_{0}} \min _{1 \leq j \leq d}\left\{m_{j}^{2} L_{j}^{2}\right\}-1>0 .
\end{aligned}
$$

For $\boldsymbol{k} \in \boldsymbol{F}_{\boldsymbol{M}} \backslash \boldsymbol{F}_{\boldsymbol{m}}$, define the $\mu_{\boldsymbol{k}}^{*}>0$ satisfying (23) by

$$
\mu_{\boldsymbol{k}}^{*}:=\frac{2}{3 \lambda_{0}}\left(k_{1}^{2} L_{1}^{2}+\cdots+k_{d}^{2} L_{d}^{2}\right)-1
$$

Therefore, we get that for all $\lambda \in\left[\lambda_{0}-\left|\Delta_{\lambda}\right|, \lambda_{0}+\left|\Delta_{\lambda}\right|\right]$ and for $\boldsymbol{k} \in \boldsymbol{F}_{\boldsymbol{M}} \backslash \boldsymbol{F}_{\boldsymbol{m}}$, we have that $\left|\mu_{\boldsymbol{k}}(\lambda)\right| \geq \mu_{\boldsymbol{k}}^{*}>0$. One can now define the $Y^{(0)}, Y^{(1)}, Y^{(2)}, Y^{(3)}$ and $Y^{(4)}$ given respectively by (24), (25), (26), (27) and (28).

We need to define the $d_{\boldsymbol{k}}^{(1,0)}, d_{\boldsymbol{k}}^{(1,1)}, d_{\boldsymbol{k}}^{(1,2)}$ and $d_{\boldsymbol{k}}^{(1,3)}$ given respectively by (32), (41), (42) and (43). Note that $d_{\boldsymbol{k}}^{(1,0)}$ is given directly by (32). For the others, let

$$
\begin{aligned}
d_{\boldsymbol{k}}^{(1,1)} & :=\frac{1}{\lambda_{0}^{2}}\left(k_{1}^{2} L_{1}^{2}+\cdots+k_{d}^{2} L_{d}^{2}\right) \frac{1}{\omega_{\boldsymbol{k}}^{s}} \\
d_{\boldsymbol{k}}^{(1,2)} & :=\frac{2}{\lambda_{0}^{3}}\left(k_{1}^{2} L_{1}^{2}+\cdots+k_{d}^{2} L_{d}^{2}\right) \frac{1}{\omega_{\boldsymbol{k}}^{s}} \\
d_{\boldsymbol{k}}^{(1,3)} & :=\mu_{\boldsymbol{k}}^{(3)} \frac{1}{\omega_{\boldsymbol{k}}^{s}}=\frac{96}{\lambda_{0}^{4}}\left(k_{1}^{2} L_{1}^{2}+\cdots+k_{d}^{2} L_{d}^{2}\right) \frac{1}{\omega_{\boldsymbol{k}}^{s}} .
\end{aligned}
$$

The other quantities needed are $\bar{A}$ and $\dot{A}$ given by (33) and (34) respectively; $Z_{\boldsymbol{k}}^{(1,0)}, Z_{\boldsymbol{k}}^{(1,1)}$, $Z_{\boldsymbol{k}}^{(1,2)}, Z_{\boldsymbol{k}}^{(2,0)}, Z_{\boldsymbol{k}}^{(2,1)}$ and $Z_{\boldsymbol{k}}^{(3,0)}$ given respectively by (35), (36), (37), (38), (39) and (40); and the bound $\tilde{\mu}_{M}$ given by (44). Defining

$$
\tilde{\mu}_{M}:=\frac{2}{3 \lambda_{0}} \min _{1 \leq j \leq d}\left\{M_{j}^{2} L_{j}^{2}\right\}-1>0,
$$

one has that

$$
\left|\mu_{\boldsymbol{k}}(\lambda)\right| \geq \tilde{\mu}_{\boldsymbol{M}}
$$

for all $\boldsymbol{k} \notin \boldsymbol{F}_{\boldsymbol{M}}$ and for all $\lambda \in\left[\lambda_{0}-\left|\Delta_{\lambda}\right|, \lambda_{0}+\left|\Delta_{\lambda}\right|\right]$.

Remark 4.1. Recalling (47) and letting $\left|\Delta_{\lambda}\right|=0$, one has that

$$
\tilde{p}_{M}(r, 0)=\frac{3 \tilde{\alpha}_{M}^{(3)}}{\tilde{\mu}_{M}}\left(\bar{A}^{2}+2 \bar{A} r+r^{2}\right)-1
$$

always has two distinct real roots, since its discriminant equals to $\frac{12 \tilde{\alpha}_{M}^{(3)}}{\tilde{\mu}_{M}}>0$. Hence, the only way we could fail to find a positive $r$ such that $\tilde{p}_{M}(r, 0)<0$ is if $\frac{3 \bar{A}^{2} \tilde{\alpha}_{M}^{(3)}}{\tilde{\mu}_{M}}-1 \geq 0$. In practice, before starting the rigorous numerical computations of the radii polynomials, we check if

$$
\frac{3 \bar{A}^{2} \tilde{\alpha}_{M}^{(3)}}{\tilde{\mu}_{M}}<1 .
$$

If condition (57) is not satisfied, we a priori know that the validation will fail. Hence, we need to increase the value of $\tilde{\mu}_{\boldsymbol{M}}$, which can be done by increasing $\boldsymbol{M}$. 


\subsection{Algorithm for the rigorous computation of global smooth branches of equilibria for the Cahn-Hilliard equation}

Algorithm 4.2. To compute rigorous global smooth branches of equilibria of the threedimensional Cahn-Hilliard equation (1) on the parameter range $\left[\lambda_{\min }, \lambda_{\max }\right]$, we proceed as follows.

1. Choose a minimum step-size $\Delta_{\min }>0$ and set the maximum step-size $\Delta_{\max }=\frac{\lambda_{\max }}{2}$. Note that with this choice, inequality (52) will always be verified. Initiate a decay rate $\boldsymbol{s}=\left(s_{1}, s_{2}, s_{3}\right)$, a projection dimension $\boldsymbol{m}=\left(m_{1}, m_{2}, m_{3}\right)$, a computational parameter $\boldsymbol{M}=\left(M_{1}, M_{2}, M_{3}\right)$, the initial parameter value $\lambda_{0}=\lambda_{\text {min }}$, an initial step size $\Delta_{\lambda}$ such that $\left|\Delta_{\lambda}\right| \in\left[\Delta_{\min }, \Delta_{\max }\right]$, a temporary step size $\Delta_{\lambda}^{0}=0$, an initial predictor $\hat{x}_{\boldsymbol{F}_{\boldsymbol{m}}}$ of $f^{(\boldsymbol{m})}\left(x_{\boldsymbol{F}_{\boldsymbol{m}}}, 0\right)$ and an initial radius $r_{0}=0$.

2. Calculate the analytic estimates $\alpha_{\boldsymbol{k}}^{(3)}, \tilde{\alpha}_{\boldsymbol{M}}^{(3)}$ and $\varepsilon_{\boldsymbol{k}}^{(3)}$, for $\boldsymbol{k} \in \boldsymbol{F}_{\boldsymbol{M}}$, using the theory from [11]. Initiate $B_{0}=B\left(\hat{\bar{x}}, r_{0}\right)$, where $\hat{\bar{x}}:=\left(\hat{x}_{\boldsymbol{F}_{\boldsymbol{m}}}, 0_{\boldsymbol{I}_{\boldsymbol{m}}}\right)$.

3. With a Newton-like iterative scheme, find near $\hat{x}_{\boldsymbol{F}_{\boldsymbol{m}}}$ an approximate solution $\bar{x}_{\boldsymbol{F}_{\boldsymbol{m}}}$ of $f^{(\boldsymbol{m})}\left(\bar{x}_{\boldsymbol{F}_{\boldsymbol{m}}}, \lambda_{0}\right)=0$. Calculate an approximate solution $\dot{x}_{\boldsymbol{F}_{\boldsymbol{m}}}$ of $D f^{(\boldsymbol{m})}\left(\bar{x}_{\boldsymbol{F}_{\boldsymbol{m}}}, \lambda_{0}\right) \dot{x}_{\boldsymbol{F}_{\boldsymbol{m}}}+$ $\frac{\partial f^{(\boldsymbol{m})}}{\partial \lambda}\left(\bar{x}_{\boldsymbol{F}_{\boldsymbol{m}}}, \lambda_{0}\right)=0$. Compute $\bar{A}, \dot{A}$ from (33), (34) and $\tilde{\mu}_{\boldsymbol{M}}$ given by (56). Verify that conditions (57) is satisfied. If not, increase $\boldsymbol{M}$ component-wise and return to Step 2.

4. Compute, using interval arithmetic, the coefficients of the radii polynomials $p_{\boldsymbol{k}}$, for $\boldsymbol{k} \in \boldsymbol{F}_{\boldsymbol{M}}$, given by (45) and (46) and $\tilde{p}_{\boldsymbol{M}}$ given by (47).

5. Defining

$$
\mathcal{I}_{0}:=\left\{r \geq 0 \mid p_{\boldsymbol{k}}(r, 0)<0 \text { for all } \boldsymbol{k} \in \boldsymbol{F}_{\boldsymbol{M}} \text { and } \tilde{p}_{\boldsymbol{M}}(r, 0)<0\right\},
$$

compute using interval arithmetic, $0<r_{1}^{-}<r_{1}^{+}$such that $\left\{r_{1}^{-}, r_{1}^{+}\right\} \subset \mathcal{I}_{0}$. In practice, we compute numerically $\inf \left(\mathcal{I}_{0}\right)$ and $\sup \left(\mathcal{I}_{0}\right)$, and we define $r_{1}^{-}=\inf \left(\mathcal{I}_{0}\right)+\delta^{-}$and $r_{1}^{+}=\sup \left(\mathcal{I}_{0}\right)-\delta^{+}$, for some small $\delta^{-}, \delta^{+}>0$. Consider $B_{1^{-}}:=B\left(\bar{x}, r_{1}^{-}\right)$and $B_{1+}:=B\left(\bar{x}, r_{1}^{+}\right)$, where $\bar{x}=\left(\bar{x}_{\boldsymbol{F}_{m}}, 0_{\boldsymbol{I}_{\boldsymbol{m}}}\right)$. Using Remark 2.6, verify that $B_{0} \subset B_{1^{+}}$or $B_{1}-\subset B_{0}$.

6. Calculate numerically

$$
\mathcal{I}=\left[I_{-}, I_{+}\right]:=\left\{r \geq 0 \mid p_{\boldsymbol{k}}\left(r, \Delta_{\lambda}\right)<0 \text { for all } \boldsymbol{k} \in \boldsymbol{F}_{\boldsymbol{M}} \text { and } \tilde{p}_{\boldsymbol{M}}\left(r, \Delta_{\lambda}\right)<0\right\} .
$$

- If $\mathcal{I}=\varnothing$ then go to Step 8 .

- If $\mathcal{I} \neq \varnothing$ then let $r=\frac{I_{-}+I_{+}}{2}$. If, computing with interval arithmetic, one can verify that $p_{\boldsymbol{k}}\left(r, \Delta_{\lambda}\right)<0$ for all $\boldsymbol{k} \in \boldsymbol{F}_{\boldsymbol{M}}$ and $\tilde{p}_{\boldsymbol{M}}\left(r, \Delta_{\lambda}\right)<0$ then go to Step 7 ; else go to Step 8.

7. Update $\Delta_{\lambda}^{0} \leftarrow \Delta_{\lambda}$ and $r_{0} \leftarrow r$. If $\frac{10}{9} \Delta_{\lambda} \leq \Delta_{\max }$ then update $\Delta_{\lambda} \leftarrow \frac{10}{9} \Delta_{\lambda}$ and go to Step 6; else go to Step 9.

8. If $\Delta_{\lambda}^{0}>0$ then go to Step 9; else if $\frac{9}{10} \Delta_{\lambda} \geq \Delta_{\min }$ then update $\Delta_{\lambda} \leftarrow \frac{9}{10} \Delta_{\lambda}$ and go to Step 6; else go to Step 10.

9. The continuation step has succeeded. Store, for future reference, $\bar{x}_{\boldsymbol{F}_{\boldsymbol{m}}}, \dot{x}_{\boldsymbol{F}_{\boldsymbol{m}}}, r_{0}, \lambda_{0}$ and $\Delta_{\lambda}^{0}$. Determine $\lambda_{1}$ approximately equal to, but interval arithmetically less than, $\lambda_{0}+\Delta_{\lambda}^{0}$. Make the updates $\lambda_{0} \leftarrow \lambda_{1}, \Delta_{\lambda} \leftarrow \Delta_{\lambda}^{0}, \hat{x}_{\boldsymbol{F}_{m}} \leftarrow \bar{x}_{\boldsymbol{F}_{m}}+\Delta_{\lambda}^{0} \dot{x}_{\boldsymbol{F}_{m}}$ and $\Delta_{\lambda}^{0} \leftarrow 0$. Update $B_{0} \leftarrow B\left(\hat{\bar{x}}, r_{0}\right)$ and go to Step 3 for the next continuation step. 
10. The continuation step has failed. Either decrease $\Delta_{\min }$ and return to Step 8; or increase some of the components of $\boldsymbol{M}$ and return to Step 4; or increase some of the components of $\boldsymbol{m}$ and return to Step 3. Alternatively, terminate the algorithm unsuccessfully at $\lambda=\lambda_{0}$

\subsection{Proof of Theorem 1.1}

Proof. The proof is computer assisted, using Algorithm 4.2. We initialize the algorithm with $\boldsymbol{s}=(2,2,2), \boldsymbol{m}=\left(m_{1}, m_{2}, m_{3}\right)=(8,8,8)$, and $\boldsymbol{M}=\left(3 m_{1}-2,3 m_{2}-2,3 m_{3}-2\right)$. We numerically find the initial points in each of the branches to initialize the algorithm. When the continuation step fails (Step 10), we increase each component of $\boldsymbol{m}$ by 1, update $\boldsymbol{M}=\left(3 m_{1}-2,3 m_{2}-2,3 m_{3}-2\right)$, and return to Step 3. Algorithm 4.2 terminates successfully for each of the branches on the diagram of Figure 1.

\section{A Results for Cahn-Hilliard in 2D}

In this section we present the results of computations for the two-dimensional Cahn-Hilliard equation. As before, for each segment of curve in the diagram of Figure 4 we have existence and local uniqueness of solutions for each value of the parameter for $c=0$.

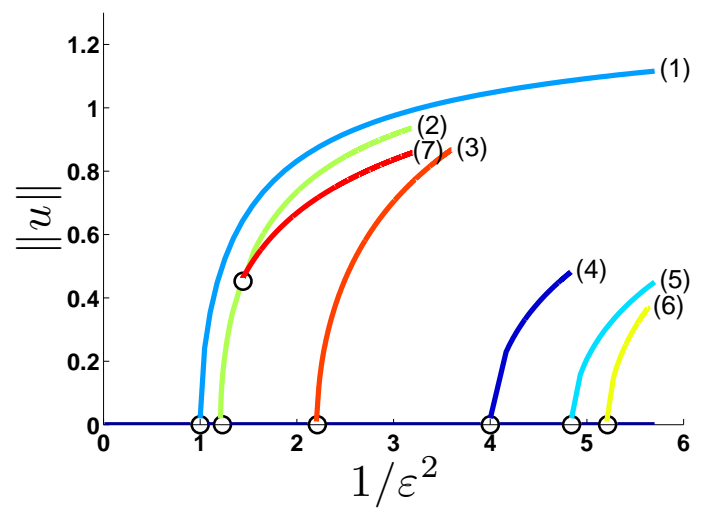

Figure 4: Smooth branches of equilibria for the Cahn-Hilliard equation posed on the twodimensional rectangular domain $\Omega=[0, \pi] \times[0, \pi / 1.1]$. Each solution is proved to lie in a ball, given by (10), of radius $r \approx 10^{-12}$ around the computed numerical approximation. Note that the apparent bifurcations are not proved. The proof of existence and smoothness for each of the branches starts shortly after the apparent bifurcation. More precisely, the bifurcations from the trivial solution occur at $\lambda=1 / \epsilon^{2} \approx 1,1.21,2.21,4,4.84$, and 5.21, and the apparent bifurcation from branch $(2)$ occur at $\lambda \approx 1.4374$. The proof of existence and smoothness for the branches (1), (2), (3), (4), (5), (6), and (7) are performed for the parameter sets $\Lambda^{(1)}=[1.0003,5.7], \Lambda^{(2)}=[1.2103,1.4087] \cup[1.4887,3.1864], \Lambda^{(3)}=$ $[2.2019,3.5986], \Lambda^{(4)}=[4.0012,4.8340], \Lambda^{(5)}=[4.8414,5.7], \Lambda^{(6)}=[5.2046,5.6318]$, and $\Lambda^{(7)}=[1.4401,3.1966]$, respectively. 


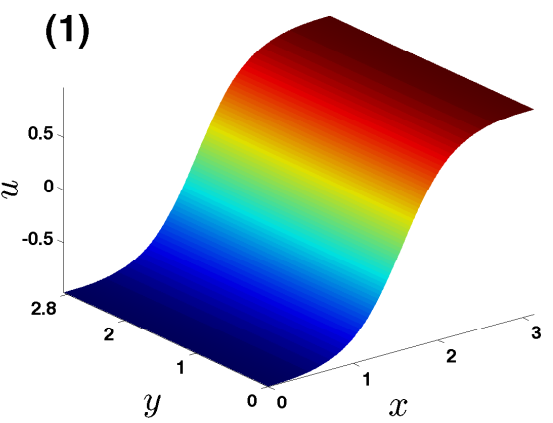

(2)
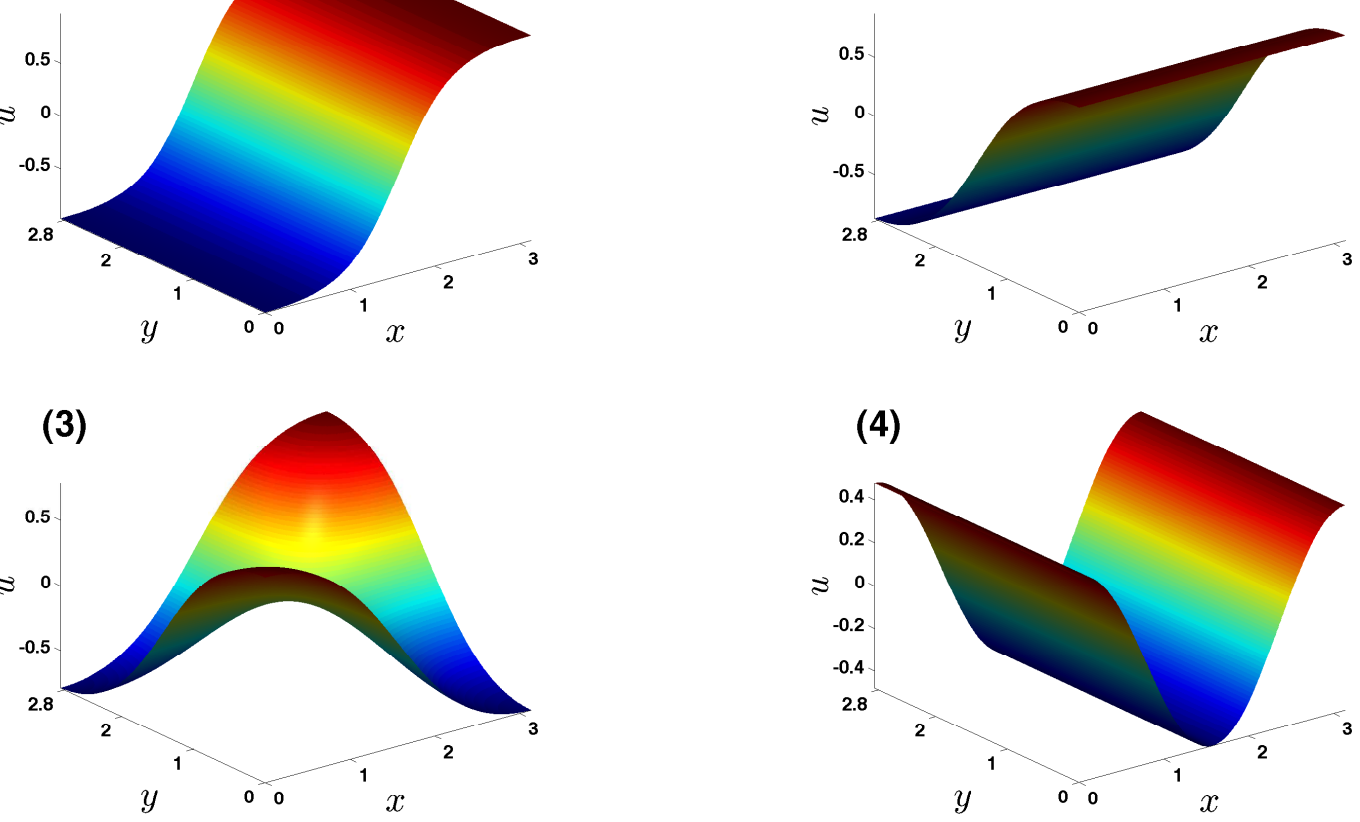

(5)
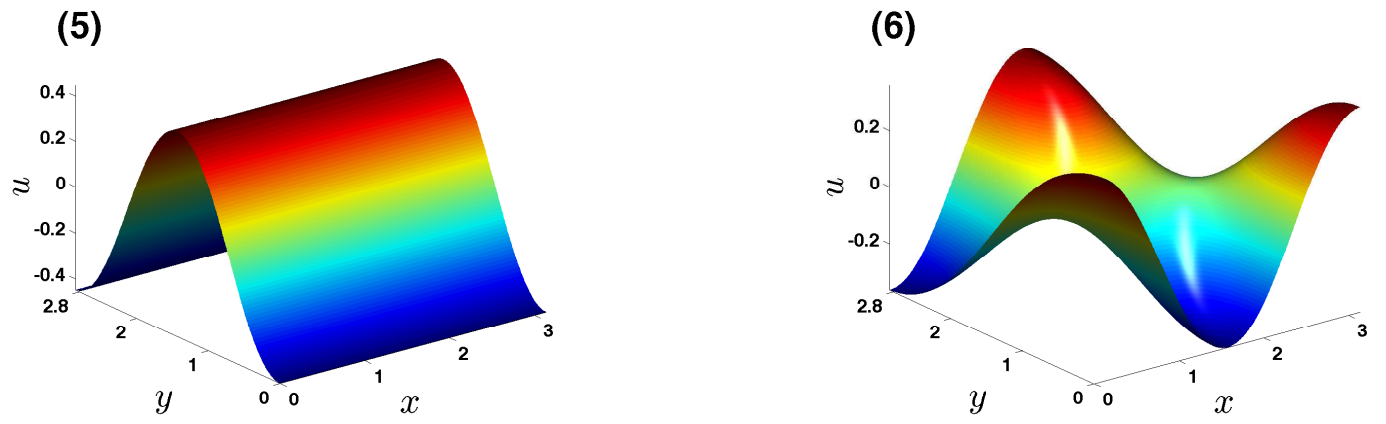

\section{(7)}

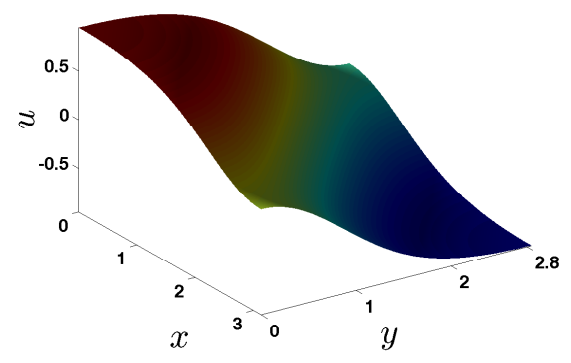

Figure 5: Solutions for the Cahn-Hilliard equation in 2D. The solutions corresponding to the last point of the branches in the diagram of Figure 4 are plotted. The labels (1), (2), (3), (4), (5), (6) and (7) correspond to the respective branches in Figure 4. 


\section{References}

[1] J. B. VAN DEN BERG AND J.-P. Lessard, Chaotic braided solutions via rigorous numerics: chaos in the Swift-Hohenberg equation, SIAM Journal on Applied Dynamical Systems, 7(3):988-1031, (2008).

[2] J. B. van den Berg, J.-P. Lessard and K. Mischaikow, Global smooth solution curves using rigorous branch following, Mathematics of Computation, 79 (271), 15651584, (2010).

[3] B. Breuer, P.J. McKenna and M. Plum, Multiple solutions for a semilinear boundary value problem: a computational multiplicity proof, Journal of Differential Equations, 195, no. 1, pp. 243-269, (2003).

[4] S. M. Allen and J. W. Cahn, A microscopic theory for antiphase boundary motion and its application to antiphase domain coarsening, Acta Metall., 27, pp. 1084-1095, (1979).

[5] J. W. Cahn and J. E. Hilliard, Free energy of a nonuniform system I. Interfacial free energy, Journal of Chemical Physics, 28, pp. 258-267, (1958).

[6] J. W. CAHn, Free energy of a nonuniform system II. Thermodynamic Basis, Journal of Chemical Physics, 30, pp. 1121-1124, (1959).

[7] J. W. Cahn and J. E. Hilliard, Free energy of a nonuniform system III. Nucleation in a Two-Component Incompressible Fluid, Journal of Chemical Physics, 31, pp. 688$699,(1959)$.

[8] S. N. Chow And J. K. Hale, Methods of bifurcation theory, volume 251 of Grundlehren der Mathematischen Wissenschaften [Fundamental Principles of Mathematical Science], Springer-Verlag, New York, 1982.

[9] S. Day, Y. Hiraoka, K. Mischaikow and T. Ogawa, Rigorous numerics for global dynamics: a study of the Swift-Hohenberg equation, SIAM Journal on Applied Dynamical Systems, 4(1):1-31 (electronic), (2005).

[10] S. Day, J.-P. Lessard and K. Mischaikow, Validated continuation for equilibria of PDEs, SIAM Journal on Numerical Analysis, 45(4):1398-1424, (2007).

[11] M. Gameiro and J.-P. Lessard, Analytic estimates and rigorous continuation for equilibria of higher-dimensional PDEs, Journal of Differential Equations, 249 (9): 2237$2268,(2010)$.

[12] M. Gameiro, J.-P. Lessard and K. Mischaikow, Validated continuation over large parameter ranges for equilibria of PDEs, Mathematics and Computers in Simulation, 79(4): 1368-1382, (2008).

[13] J. G. Heywood, W. Nagata and W. Xie, A numerically based existence theorem for the Navier-Stokes equations, J. Math. Fluid Mech. 1, no. 1, 5-23, (1999).

[14] H. B. KeLLER, Lectures on numerical methods in bifurcation problems, Tata Institute of Fundamental Research Lectures on Mathematics and Physics, 79, Springer-Verlag, Berlin, 1987. 
[15] M. Kim, M. T. Nakao, Y. Watanabe and T. Nishida, A numerical verification method of bifurcating solutions for 3-dimensional Rayleigh-Bnard problems, $\mathrm{Nu}$ merische Mathematik, 111, no. 3, 389-406, (2009).

[16] J.-P. LEssard, Recent advances about the uniqueness of the slowly oscillating periodic solutions of Wright's equation, Journal of Differential Equations, 248 (5): 992-1016, (2010).

[17] S. Maier-Paape, K. Mischaikow and T. Wanner, Structure of the attractor of the Cahn-Hilliard equation on a square, Internat. J. Bifur. Chaos Appl. Sci. Engrg. 17, no. 4, 1221-1263, (2007).

[18] S. Maier-Paape, U. Miller, K. Mischaikow and T. Wanner, Rigorous numerics for the Cahn-Hilliard equation on the unit square, Rev. Mat. Complut. 21, no. 2, 351$426,(2008)$.

[19] M. T. NAKaO, Numerical verification methods for solutions of ordinary and partial differential equations, Numer. Funct. Anal. Optim. 22, no. 3-4, 321-356, (2001).

[20] M. T. Nakao, K. Hashimoto and Y. Watanabe, A numerical method to verify the invertibility of linear elliptic operators with applications to nonlinear problems, Computing, 75, no. 1, 1-14, (2005).

[21] M. T. Nakao, N. Yamamoto and Y. Nishimura, Numerical verification of the solution curve for some parametrized nonlinear elliptic problem. Proceedings of Third China-Japan Seminar on Numerical Mathematics, 238-245, Science Press, Beijing, 1998.

[22] M. Plum, Explicit $H_{2}$-estimates and pointwise bounds for solutions of second-order elliptic boundary value problems, J. Math. Anal. Appl. 165, no. 1, 36-61, (1992).

[23] M. Plum, Existence and enclosure results for continua of solutions of parameterdependent nonlinear boundary value problems, J. Comput. Appl. Math. 60, no. 1-2, 187-200, (1995).

[24] N. Yамамото, A numerical verification method for solutions of boundary value problems with local uniqueness by Banach's fixed-point theorem, SIAM Journal on Numerical Analysis, 35, pp. 2004-2013, (1998).

[25] P. ZGLICZYŃSKI, Rigorous numerics for dissipative partial differential equations. II. Periodic orbit for the Kuramoto-Sivashinsky PDE - a computer-assisted proof, Found. Comput. Math. 4, no. 2, 157-185, (2004).

[26] P. ZgLiczyŃski and K. Mischaikow, Rigorous numerics for partial differential equations: The Kuramoto-Sivashinsky equation, Found. Comput. Math., 1, pp. 255-288, (2001). 